\title{
Cryostat Filling Limitations for Proposed Ar Dewar Pressure Increase
}

\author{
3740.512-EN-321 \\ July 23,1991 \\ J. Wu/K. Dixon \\ Revised October 17, 1991

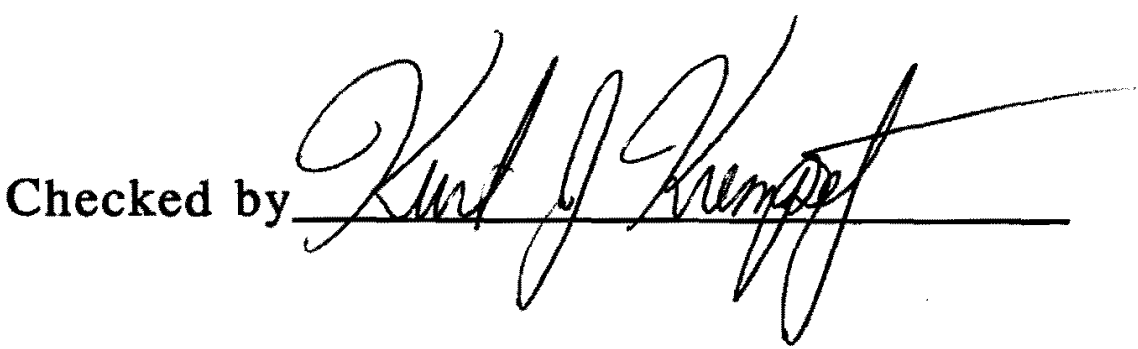




\section{Cryostat Filling Limitations for Proposed} Ar Dewar Pressure Increase

In order to significantly decrease the amount of time required to fill the cryostats, it is desired to raise the setpoint of the "operating" relief valve on the argon storage dewar to 20 psig from its existing 16 psig setting. This additional pressure increases the flow to the cryostats and will overwhelm the relief capacity if the temperature of the modules within these vessels is warm enough. Using some conservative assumptions and simple calculations within this note, the maximum average temperature that the modules within each cryostat can be at prior to filling from the storage dewar with liquid argon is at least $290 \mathrm{~K}$.

\section{Some Assumptions Used in the Analysis}

1. Pressure in the argon storage dewar is at 20 psig.

2. The flow to the ECN cryostat is the most hazardous due to greater limitations on venting (see attached calculations).

3. The maximum flow of argon to the cryostat is $\mathbf{1 2 . 3} \mathrm{gpm}$ (see attached calculations).

4. Gaseous nitrogen is concurrently flowing in the vent piping at a rate of $4861 \mathrm{lb} / \mathrm{hr}$, this is derived from both ECS and CC cooling at their maximum rate and ECN condenser attempting to maintain pressure at its maximum.

5. Mixture mass flows are at the maximum at junction of relief devices on $\mathrm{ECN}\left(\mathrm{gN}_{2}\right.$ mass flow actually increases gradually at junctions toward the ECS).

6. The temperature increase in the vent piping is negligible (large majority of piping is insulated).

7. All flows are treated as incompressible fluids (max. Mach No. $=0.2$ ). 
8. Temperature of the gaseous nitrogen prior to mixing in the vent manifold is $84 \mathrm{~K}$, saturated property at 2 atm.

9. Flow equations apply to weight-averaged mixture densities and viscosities.

10. All liquid argon flashes to the bulk module temperature in the cryostat prior to entering the piping.

\section{Explanation of Methodology}

The basic purpose of the spreadsheet was to provide a complete model so that the maximum bulk temperature of the modules (line 2) could be determined. The maximum flow of argon to the ECN (line 3) was calculated separately and included after the spreadsheet. A number was picked as a guess for the bulk temperature (line 2). Then a number was picked as a guess for the percent of mass flow to the relief valve (line 18). The actual flow through the relief valve (line 98) was determined using the total flow and the percent of flow to the relief valve. The pressure drops across the inlet and outlets of the relief devices were then calculated. This allowed the calculation of the pressure drops across the relief valve and rupture disk. The various properties of argon were taken from tables at the bulk temperature of the modules.

The section " $\Delta \mathrm{P}$ Across Relief Valve" calculated the maximum theoretical flow of argon through the relief valve (line 108). Then the theoretical percent of relief flow (line 109) was calculated based on the theoretical relief valve flow divided by the total flow through both the relief valve and rupture disk. The number guessed for the percent of flow to the relief valve (line 18) was then adjusted by iteration until it was close to, but not greater than, the theoretical value (line 109). At this point, the relief valve was operating near its full capacity, which could be checked by noting that the actual flow (line 98) was close to the maximum theoretical flow (line 108). 
The next step was to examine the section " $\Delta \mathrm{P}$ Across Rupture Disk". All of the total argon flow not going though the relief valve would be flowing through the rupture disk. In order to insure that the rupture disk could handle the flow, the maximum theoretical rupture disk flow (line 181) was calculated, and compared to the actual flow (line 182). The actual flow had to be under the maximum theoretical flow, but should be close to the maximum value to obtain the highest total flow. In the case that the actual flow was calculated to be higher than the maximum theoretical flow, the bulk temperature was lowered. Using the new temperature, the first set of iterations was repeated to determine the percent of flow to the relief valve, and the rupture disk flow was compared again. The temperature was lowered through iteration until an acceptable value was found.

Note that the sections on pressure drops were only needed to calculate inlet and outlet pressures for the relief valve and rupture disk. Other sections calculated the changes in various properties of the argon at certain points. Each time the temperature was changed, the values for density and viscosity were changed to reflect the new temperature. The maximum flow of nitrogen from the condensers was also accounted for, since it had an effect on the pressure drops of the outlets of the relief devices.

\section{Notes on Maximum Module Temperature Calculation}

* $>$ means that this value is to be re-entered each time the bulk module temperature is changed.

$>$ means that this value is a number, not a formula, but should only be entered once, i.e., it doesn't need to change with the temperature.

(conv.) means that this value is the same as a previous value, but converted to different units.

EN-263, Russ Rucinski, should be referred to for pressure drop calculations. 


\section{General Procedure:}

In the first section, "Conversion of Liquid to Gas at Module Temp.," enter the bulk temperature of the modules. This is also the temperature that will be used for pressure drops in the relief and rupture disk inlets, and for the relief devices themselves. Enter the gas density at 2.2 bars and 2.4 bars, and at the bulk temperature, so that the density at the cryostat pressure can be calculated. Enter some percent of mass flow to the relief valve. This will be used to assume some mass flow to each relief device for pressure drop calculations. It will be adjusted by iteration later.

In the next section, " $\Delta \mathrm{P}$ Across Relief Valve Inlet," enter the viscosity at 2.4 bars (or 2.375 bars for more accuracy) and the bulk temperature. The rest of the section is calculated.

The next section, " $\Delta \mathrm{P}$ Across Rupture Disk Inlet," needs no entries, since it assumes the same gas properties as the previous section.

The section, " $\Delta \mathrm{P}$ Across Relief Valve Outlet" requires the gas density, and the viscosity at an verge pressure of 2 bars and at the bulk temperature. This just accounts for the drop in pressure to about 1.5 bars. If more accuracy is required, the new pressure could be calculated by adding the common outlet pressure drops to atmospheric pressure.

The " $\Delta \mathrm{P}$ Across Rupture Disk Outlet" section is completely calculated, based on the assumption that the gas properties remain the same as for the relief valve outlet.

The next section, "Change in Gas at Common Outlet to Outside" reflects the change in properties of the fluid at the junction of the relief device outlets due to the mixing of argon from the relief devices and nitrogen from the condensers.

The " $\Delta \mathrm{P}$ Across Relief Valve" is completely calculated (ref.1, 3). The specific heat ratio, $k$, has been determined using the $C_{p}$ and $C_{v}$ at the correct temperature and pressure. Also, the flowing temperature is converted from the original bulk temperature, to the equivalent Rankine 
temperature. The basic purpose of this section is to compare the "Theoretical Percent of Relief Flow" to the actual percent that was entered in section 1. Since the theoretical percent of the relief flow is the maximum flow possible at the given inlet and outlet parameters, this number should be checked such that it does not fall below the "guessed" percentage in the first section of calculations.

The " $\Delta P$ Across Common Outlet to Platform" is calculated based on the properties from the "Change in Gas..." section. Also, all pressure drop calculations are based on a equation which relates the friction factor, $f$, to the Reynolds number and the relative roughness, e/D. The "Friction Factor Guess" is based on an equation in Introduction to Fluid Mechanics (ref.1) and that value is used in another equation in the same reference to find the actual friction factor. Calculations to determine equivalent lengths and relative roughness were based on dimensions from sketches and drawings of the ECN piping and platform manifold.

The section on the " $\Delta P$ from Platform Bayonet to Outside" is completely calculated like the previous section, but with a different diameter and equivalent length.

The "Summation of Equivalent $\Delta P$ " is basically a summary of the pressure drops, where the "Rupture Disk Pressure Drop" is calculated based on the three relief valve values, and the rupture disk inlet and outlet values.

The " $\Delta P$ Across Rupture Disk Device" is calculated like the relief valve. The complete equation for the "Gas Flow Constant for Subsonic Flow (C1)" is found in reference 2 . The specific heat ratio, $k$, was adjusted according to the actual pressure and temperature. In both the relief valve and the rupture disk, the outlet pressure should be compared to the critical pressure, which it must exceed for the flow to be subsonic. In all cases analyzed, the flow was subsonic. 


\section{Conclusions and Recommendations}

The average temperature of the module mass for any of the three cryostats can be as high as $290 \mathrm{~K}$ prior to filling that particular cryostat. This should not be confused with the average temperature of a single type or location which is useful in protecting the modules-not necessarily the vessel itself. A few modules of each type and at different elevations should be used in an average which would account for the different weights of each module. Note that at $290 \mathrm{~K}$, the actual flow of argon through the relief valve and the rupture disk was under the maximum theoretical flows for each relief device. This means that the bulk temperature could actually have been raised to flow argon through the reliefs at their maximum capacity. Therefore, the temperature of $290 \mathrm{~K}$ is a conservative value for the calculated flow rate of $12.3 \mathrm{gpm}$.

Safeguards in addition to and used in conjunction with operating procedures shall be implemented in such a way so that the above temperature limitation is not exceeded and such that it is exclusive of the programmable logic controller (PLC). One suggestion is using a toggle switch for each cryostat mounted in the PLC I/O box which would maintain control of the signals to open the cold fill valves of each cryostat.

With the safeguards in place while carefully monitoring the temperatures during a cooldown cycle in each cryostat, the set pressure in the argon storage dewar can safely be increased to 20 psig. 


\section{References}

1. Introduction to Fluid Mechanics, 3rd Ed., Robert W. Fox, Alan T. McDonald, JohnWiley\&Sons, 1985.

2. “Fike Technical Bulletin TB 8102, Rupture Disk Sizing", Fike Metal Products Corp.

3. "Catalog 1900-Series 90 Safety Relief Valves", Anderson, Greenwood \& Co., 1980.

4. "D $\varnothing \mathrm{CC}$ Pressure Vessel and Vacuum Vessel Safety Note", D $\varnothing$ Engineering Note \#3740-EN-263, R. Rucinski/R. Luther, Nov., 1990. 


\begin{tabular}{|c|c|c|c|c|}
\hline & $\mathbf{A}$ & $\mathbf{B}$ & $\overline{\mathbf{C}}$ & D \\
\hline 1 & Conversion of Liquid to Gas at Module Temp. & & & Units \\
\hline 3 & $>$ Max. Flow of Liquid Argon to Cryostat & 12.3 & 12.3 & gpm \\
\hline 4 & $>$ Pressure in Cryostat & 19.75 & 19.75 & psig \\
\hline 6 & $>$ IAr Density@ 2.2 bars & 1.342421 & 1.34 & $g / c c$ \\
\hline 7 & $>$ IAr Density @ 2.4 bars & 1.335861 & 1.34 & $g / c c$ \\
\hline 8 & IAr Density @ 2.375 bars & $=(B 5-2.2) / 0.2^{*}(B 7-B 6)+B 6$ & 1.337 & $g / c c$ \\
\hline 11 & gAr Density @ 2.375 \& Tomp. & $=(\mathrm{B} 5-2.2) / 0.2^{*}(\mathrm{~B} 10-\mathrm{B} 9)+\mathrm{B} 9$ & 3.945 & $\mathrm{mg} / \mathrm{cc}$ \\
\hline 12 & gAr Density @ 2.375 bars (conv.) & $=B 11 / 1000^{*} 62.428$ & 0.246 & $1 \mathrm{bm} / \mathrm{ft}^{\wedge} 3$ \\
\hline 13 & Maximum Flow of Argon Gas & $=B 3^{*} \mathrm{~B} 8 / \mathrm{B} 11 * 1000$ & 4167 & $\mathrm{gpm}$ \\
\hline 14 & Maximum Flow of Argon Gas (conv.) & $=B 13 * 0.13368$ & 557 & $\operatorname{cfm}$ \\
\hline 15 & Air Equivalent Flow @STP & $=6.32^{*} \mathrm{~B} 17^{*} 356 / \mathrm{B} 16^{*} \mathrm{SQRT}\left(\mathrm{B} 99 /\left(520^{*} \mathrm{~B} 17^{\prime *} 28.97\right)\right)$ & 1443 & scfm air \\
\hline 16 & Specific Heat Constant, C, for Ar & $=520^{\circ} \operatorname{SQRT}\left(B 168^{\circ}(2 /(B 168+1))^{\wedge}((B 168+1) /(B 168-1))\right)$ & 378 & \\
\hline 17 & Max. Mass Flow of Argon Gas & $=\mathrm{B} 14^{*} \mathrm{~B} 12^{*} 60$ & 8232 & $\mathrm{lbm} / \mathrm{hr}$ \\
\hline 23 & $>$ Equivalent Length & 38 & 38 & ft \\
\hline 24 & Ar Gas Density @ 2.375 \& Temp. & $=(\mathrm{B} 5-2.2) / 0.2^{*}(\mathrm{~B} 10-\mathrm{B} 9)+\mathrm{B} 9$ & 3.945 & $\mathrm{mg} / \mathrm{cc}$ \\
\hline 25 & Ar Gas Density @ 2.375 bars (conv.) & $=B 24 / 1000^{*} 62.428$ & 0.246 & $1 \mathrm{bm} / \mathrm{ft}^{\wedge} 3$ \\
\hline 26 & > gAr Viscosity @ 2.4 bars \& Temp. & 0.0002228 & 0.0002228 & $\mathrm{~g} / \mathrm{cm}-\mathrm{s}$ \\
\hline 27 & aAr Viscosity @ 2.4 bars (conv.) & $=B 26 * 100$ & 0.02228 & centipoiso \\
\hline 28 & Max. Mass Flow to Relief Valve & $=B 17^{* B 18}$ & 3952 & $1 \mathrm{bm} / \mathrm{hr}$ \\
\hline 28 & Reynolds Number & $=6.31 * \mathrm{~B} 28 /\left(\mathrm{B} 22{ }^{*} \mathrm{~B} 27\right)$ & 453000 & \\
\hline 30 & $>$ Relative Roughness (e/D) & 0.0007 & 0.0007 & \\
\hline 31 & Friction Factor Guess & $=0.25^{*}\left(\mathrm{LOG}\left(\mathrm{B} 30 / 3.7+5.74 /\left(\mathrm{B} 29^{\wedge} 0.9\right)\right)\right)^{\wedge}-2$ & 0.019 & \\
\hline 32 & Friction Factor & $=0.25^{\circ}\left(\mathrm{LOG}\left(\mathrm{B} 30 / 3.7+2.51 /\left(\mathrm{B} 29^{*} \mathrm{~B} 31^{\wedge} 0.5\right)\right)\right)^{\wedge}-2$ & 0.0189 & \\
\hline 33 & Pressure Drop & $=0.000000336^{\circ} B 32^{*}{\mathrm{~B} 23^{*}}^{*}\left(\mathrm{~B} 28^{\wedge} 2\right) / \mathrm{B} 25 /\left(\mathrm{B} 22^{\wedge} 5\right)$ & 1.655 & psi \\
\hline 34 & & & & \\
\hline
\end{tabular}




\section{Maximum Module Temperature Calculation}

\begin{tabular}{|c|c|c|c|c|}
\hline & A & $\mathbf{B}$ & C & D \\
\hline 35 & $\Delta \mathrm{P}$ Across Rupture Disk Inlet & & & Units \\
\hline 36 & Inner Pipe Dlameter (conv.) & $=B 37 / 12$ & 0.172 & ft \\
\hline 37 & $>$ Inner Pipe Diameter & 2.067 & 2.067 & in \\
\hline 38 & $>$ Equivalent Length & 49 & 49 & $f t$ \\
\hline 39 & gAr Density @ 2.375 \& Temp. & $=(B 5-2.2) / 0.2^{*}(B 10-B 9)+B 9$ & 3.945 & $\mathrm{mg} / \mathrm{cc}$ \\
\hline 40 & gAr Density @ 2.375 bars (conv.) & $=\mathrm{B} 39 / 1000^{*} 62.428$ & 0.246 & $\mathrm{Ibm} / \mathrm{ft}^{\wedge} 3$ \\
\hline 41 & gAr Viscosity @ 2.4 bars & $-\mathrm{B} 26$ & 0.0002228 & $\mathrm{~g} / \mathrm{cm}-\mathrm{s}$ \\
\hline 42 & gAr Viscosity @ 2.4 bars (conv.) & $=B 41 * 100$ & 0.02228 & centipoise \\
\hline 43 & Max. Mass Flow to Rupture Disk & $-B 17^{*}(1-B 18)$ & 4281 & $\mathrm{lbm} / \mathrm{hr}$ \\
\hline 44 & Reynolds Number & $=6.31^{\circ} \mathrm{B} 43 /\left(\mathrm{B} 37^{*} \mathrm{~B} 42\right)$ & 587000 & \\
\hline 45 & $>$ Relative Roughness (e/D) & 0.0009 & 0.0009 & \\
\hline 46 & Friction Factor Guess & $=0.25^{*}\left(\operatorname{LOG}\left(B 45 / 3.7+5.74 /\left(B 44^{\wedge} 0.9\right)\right)\right)^{\wedge}-2$ & 0.0198 & \\
\hline 47 & Friction Factor & $=0.25^{*}\left(\operatorname{LOG}\left(B 45 / 3.7+2.51 /\left(\mathrm{B} 44^{\circ} \mathrm{B} 46^{\wedge} 0.5\right)\right)\right)^{\wedge}-2$ & 0.0197 & \\
\hline 48 & Pressure Drop & $=0.00000336^{*} \mathrm{~B} 47^{*} \mathrm{~B} 38^{*}\left(\mathrm{~B} 43^{\wedge} 2\right) / \mathrm{B} 40 /\left(\mathrm{B} 37^{\wedge} 5\right)$ & 6.394 & psi \\
\hline 51 & & & & \\
\hline 52 & $\triangle \mathrm{P}$ Across Relief Valve Outlet & & & Units \\
\hline 53 & Inner Pipe Diameter (conv.) & $=854 / 12$ & 0.272 & ft \\
\hline 54 & $>$ Inner Pipe Diameter & 3.26 & 3.26 & in \\
\hline 55 & $>$ Equivalent Length & 51 & 51 & $f t$ \\
\hline 56 & > gAr Density @ 2.0 bar \& Temp. & 3.324 & 3.324 & $\mathrm{mg} / \mathrm{cc}$ \\
\hline 57 & gAr Density@ $@ 2.0$ bar (conv.) & $=856 / 1000^{*} 62.428$ & 0.208 & $1 \mathrm{bm} / \mathrm{ft}^{\wedge} 3$ \\
\hline 58 & > gAr Viscosity @ 2.0 bar \& Tomp. & 0.0002227 & 0.0002227 & $\mathrm{~g} / \mathrm{cm}-\mathrm{s}$ \\
\hline 59 & gAr Viscosity @ 2.0 bar (conv.) & $=858 * 100$ & 0.02227 & centipoise \\
\hline 60 & Max. Mass Flow to Relief Valve & $=\mathrm{B28}$ & 3952 & $16 \mathrm{~m} / \mathrm{hr}$ \\
\hline 61 & Reynolds Number & $-6.31^{\circ} \mathrm{B} 60 /\left(\mathrm{B} 54^{*} \mathrm{~B} 59\right)$ & 343000 & \\
\hline 62 & $>$ Relative Roughness $(e / D)$ & 0.00055 & 0.00055 & \\
\hline 63 & Friction Factor Guess & $-0.25^{\circ}\left(\mathrm{LOG}\left(\mathrm{B} 62 / 3.7+5.74 /\left(\mathrm{B} 61^{\wedge} 0.9\right)\right)\right)^{\wedge}-2$ & 0.0185 & \\
\hline 64 & Friction Factor & $=0.25^{*}\left(\operatorname{LOG}\left(B 62 / 3.7+2.51 /\left(B 61^{*} B 63^{\wedge} 0.5\right)\right)\right)^{\wedge}-2$ & 0.0183 & \\
\hline 65 & Pressure Drop & $=0.00000336^{*} \mathrm{~B} 64^{*} \mathrm{~B} 55^{*}\left(\mathrm{~B} 60^{\wedge} 2\right) / \mathrm{B} 57 /\left(\mathrm{B} 54^{\wedge} 5\right)$ & 0.642 & psi \\
\hline 66 & & & & \\
\hline
\end{tabular}




\begin{tabular}{|c|c|c|c|c|}
\hline & A & $\mathbf{B}$ & $\mathbf{C}$ & D \\
\hline 67 & $\Delta \mathrm{P}$ Across Rupture Disk Outlet & & & Units \\
\hline 68 & Inner Pipe Diameter (com.) & $=869 / 12$ & 0.18 & f t \\
\hline 69 & $>$ Inner Pipe Diameter & 2.157 & 2.157 & in \\
\hline 70 & $>$ Equivalent Length & 1.75 & 1.75 & $\mathrm{ft}$ \\
\hline 71 & gAr Density @ 2.0 bar \& Temp. & $=\mathrm{B} 56$ & 3.324 & $\mathrm{mg} / \mathrm{cc}$ \\
\hline 72 & gAr Density @ 2.0 bar (conv.) & $=B 71 / 1000^{*} 62.428$ & 0.208 & $16 m / f t^{\wedge} 3$ \\
\hline 73 & gAr Viscosity @ 2.0 bar \& Temp. & -B58 & 0.0002227 & $\mathrm{~g} / \mathrm{cm}-\mathrm{s}$ \\
\hline 74 & gAr Viscosity @2.0 bar (conv.) & $=B 73 * 100$ & 0.02227 & contipoise \\
\hline 75 & Max. Mass Flow to Rupture Disk & $=\mathrm{B} 43$ & 4281 & $\mathrm{lbm} / \mathrm{hr}$ \\
\hline 76 & Reynolds Number & $=6.31^{*} \mathrm{~B} 75 /\left(\mathrm{B} 69^{\circ} \mathrm{B} 74\right)$ & 562000 & \\
\hline 77 & $>$ Relative Roughness $(\theta / D)$ & 0.0009 & 0.0009 & \\
\hline 78 & Friction Factor Guess & $=0.25^{*}\left(\operatorname{LOG}\left(B 77 / 3.7+5.74 /\left(B 76^{\wedge} 0.9\right)\right)\right)^{\wedge}-2$ & 0.0198 & \\
\hline 78 & Friction Factor & $-0.25^{*}\left(\mathrm{LOG}\left(B 77 / 3.7+2.51 /\left(B 76^{\star} B 78^{\wedge} 0.5\right)\right)\right)^{\wedge}-2$ & 0.0197 & \\
\hline 80 & Pressure Drop & $-0.00000336^{*} \mathrm{~B} 79^{*} \mathrm{~B} 70^{*}\left(\mathrm{~B} 75^{\wedge} 2\right) / \mathrm{B} 72 /\left(\mathrm{B} 69^{\wedge} 5\right)$ & 0.219 & |psi \\
\hline 81 & & & & \\
\hline 82 & Change in Gas at Common Outlet to Outside & & & Units \\
\hline 83 & Pressure in Cryostat & $-B 4$ & 19.75 & psig \\
\hline 84 & Pressure in Cryostat (conv.) & $=(B 83 / 14.696+1)^{*} 1.01325$ & 2.375 & bars \\
\hline B5 & gAr Density @ 2.2 bars & B9 & 3.655 & $\mathrm{mg} / \mathrm{cc}$ \\
\hline 86 & gAr Density@ @ 2.4 bars & -B10 & 3.987 & $\mathrm{mg} / \mathrm{cc}$ \\
\hline 87 & gAr Density @ 2.375 \& Temp. & $=(B 84-2.2) / 0.2^{*}(B 86-B 85)+B 85$ & 3.945 & $\mathrm{mg} / \mathrm{cc}$ \\
\hline 88 & Temp. at Common Outlet & $=\left(B 2 * B 17+84^{*} \mathrm{~B} 189\right) /(\mathrm{B} 17+\mathrm{B} 189)$ & 214 & $\mathbf{K}$ \\
\hline 89 & $>$ Pressure to Calculate Density & 1.5 & 1.5 & bars \\
\hline 80 & $>$ gAr Density @ 1.5 bars \& Now Tomp. & 3.396 & 3.396 & $\mathrm{mg} / \mathrm{cc}$ \\
\hline 91 & $>$ gAr Viscosity @ 1.5 bars \& Now Temp. & 0.0001696 & 0.0001696 & $\mathrm{~g} / \mathrm{cm}-\mathrm{s}$ \\
\hline 92 & gAr Viscosity @ 1.5 bar (conv.) & $=B 91 * 100$ & 0.01696 & centipoise \\
\hline 93 & & & & \\
\hline
\end{tabular}




\section{Maximum Module Temperature Calculation}

10/17/91

\begin{tabular}{|c|c|c|c|c|}
\hline & $\bar{A}$ & $\mathbf{B}$ & $\mathbf{C}$ & $\mathbf{D}$ \\
\hline 94 & $\triangle \mathrm{P}$ Across Relief Valve & & & Units \\
\hline 95 & $>$ Critical Ratio (Pcr/P1) for Argon & 0.487 & 0.487 & \\
\hline 96 & $>$ Specific Heat Ratio (k) for Argon & $=B 168$ & 1.67 & \\
\hline 97 & $>$ Area of $2^{n} \times 3^{n}$ Relief Valve & 2.29 & 2.29 & in`2 $^{\wedge}$ \\
\hline 98 & Flow Through Relief Valve & $=828$ & 3952 & $\mathrm{lbm} / \mathrm{hr}$ \\
\hline 99 & Flowing Temperature & $=1.8^{\star} \mathrm{B} 2$ & 522 & $\operatorname{deg} A$ \\
\hline 100 & $>$ Compressibility Factor & 1 & 1 & \\
\hline 101 & $>$ Nozzle Coefficient for type $83 \mathrm{~T}$ & 0.939 & 0.939 & \\
\hline 102 & Flowing Inlat Pressure (P1) & $=\mathrm{B} 4+14.696-\mathrm{B} 33$ & 32.79 & psia \\
\hline 103 & > Molecular Weight of Argon & 39.948 & 39.95 & $\mathrm{~g} / \mathrm{mol}$ \\
\hline 104 & Critical Pressure (Pcr) & $-B 95^{*} \mathrm{~B} 102$ & 15.97 & psia \\
\hline 105 & Outlet Pressure (P2) (using delta p's) & $-14.696+\mathrm{B} 154+\mathrm{B} 133+\mathrm{B} 65$ & 27.39 & psia \\
\hline 106 & Pressure Ratio (P2"/P1) & $=\left(\mathrm{B} 102-0.55^{\star}\left((\mathrm{B} 102-\mathrm{B} 105)^{\wedge} 0.98\right)\right) / \mathrm{B} 102$ & 0.912 & \\
\hline 107 & Theoretical Factor ( $F^{*}$ ) (using $P_{2}$ ) & $=\operatorname{SQRT}\left((\mathrm{B} 96 /(\mathrm{B} 96-1))^{*}\left(\mathrm{~B} 106^{\wedge}(2 / \mathrm{B} 96)-\mathrm{B} 106^{\wedge}((\mathrm{B} 96+1) / \mathrm{B} 96)\right)\right)$ & 0.284 & \\
\hline 108 & Max. Theoretical Relief Flow (using $F^{*}$ ) & $=735^{*} \mathrm{~B} 97^{*} \mathrm{~B} 101^{*} \mathrm{~B} 102^{*} \mathrm{~B} 107^{*} \mathrm{SQRT}(\mathrm{B} 103 / \mathrm{B} 99 / \mathrm{B} 100)$ & 4072 & $1 \mathrm{bm} / \mathrm{hr}$ \\
\hline 108 & Theoretical Percent of Relief Flow & $=\mathrm{B} 108 / \mathrm{B} 17$ & 0.4947 & \\
\hline 110 & Pressure Drop Across Relief Valve & $=\mathrm{B} 102-\mathrm{B} 105$ & 5.403 & psi \\
\hline 111 & & & & \\
\hline
\end{tabular}




\section{Maximum Module Temperature Calculation}

\begin{tabular}{|c|c|c|c|c|}
\hline & A & B & C & D \\
\hline 112 & $\Delta \mathrm{P}$ Across Common Outlet to Platform Bayonet & & & Units \\
\hline 113 & $>$ Inner Pipe Diameter & 0.355 & 0.355 & $t+$ \\
\hline 114 & Inner Pipe Diameter (conv.) & $=B 113 * 12$ & 4.26 & in \\
\hline 115 & $>$ Equivalont Length & 273 & 273 & $f t$ \\
\hline 116 & gAr Donsity @ 1.5 bar \& Now Temp. & -890 & 3.396 & $\mathrm{mg} / \mathrm{cc}$ \\
\hline 117 & gAr Donsity @ 1.5 bar (conv.) & $=B 116 / 1000 * 62.428$ & 0.212 & $1 \mathrm{bm} / \mathrm{ft}^{\wedge} 3$ \\
\hline 118 & > gN2 Gas Density @ 1.5 bar \& New Temp. & 2.411028 & 2.411 & $\mathrm{mg} / \mathrm{cc}$ \\
\hline 110 & gN2 Gas Density @ 1.5 bar (conv.) & $-B 118 / 1000 * 62.428$ & 0.151 & $\mathrm{Ibm} / \mathrm{ft}^{\wedge} \mathrm{3}$ \\
\hline 120 & Gas Mixture Density @1.5 bar & $=\left(B 17^{*} B 117+B 127^{*} B 119\right) / B 128$ & 0.189 & $1 \mathrm{bm} / \mathrm{ft}^{\wedge} 3$ \\
\hline 121 & gAr Viscosity @ 1.5 bar \& Now Temp. & B91 & 0.0001696 & $\mathrm{~g} / \mathrm{cm}-\mathrm{s}$ \\
\hline 122 & gAr Viscosity @ 1.5 bar (conv.) & $=B 121 \cdot 100$ & 0.01696 & centipoise \\
\hline 123 & $>$ gN2 Viscosity @ 1.5 bar \& Now Temp. & 0.000136454 & 0.0001365 & $\mathrm{~g} / \mathrm{cm}-\mathrm{s}$ \\
\hline 124 & gN2 Viscosity @ 1.5 bar (conv.) & $=B 123 * 100$ & 0.01365 & centipoise \\
\hline 125 & Mixture Viscosity @1.5 bar & $=\left(\mathrm{B} 17^{*} \mathrm{~B} 122+\mathrm{B} 127^{*} \mathrm{~B} 124\right) / \mathrm{B} 128$ & 0.01573 & centipoise \\
\hline 126 & Max. Mass Flow of Argon Gas & $-B 17$ & 8232 & $\mathrm{Ibm} / \mathrm{hr}$ \\
\hline 127 & Max. Flow of Nitrogen Gas & $-B 189$ & 4861 & $\mathrm{Ibm} / \mathrm{hr}$ \\
\hline 128 & Mass Flow of Mixture & $-B 126+B 127$ & 13093 & $\mathrm{bm} / \mathrm{hr}$ \\
\hline 129 & Reynolds Number & $-6.31^{\circ} \mathrm{B} 128 /\left(\mathrm{B} 114^{\circ} \mathrm{B} 125\right)$ & 1230000 & \\
\hline 130 & $>$ Relative Roughness $(\theta / D)$ & 0.0004 & 0.0004 & \\
\hline 131 & Friction Factor Guess & $=0.25^{*}\left(\operatorname{LOG}\left(\mathrm{B} 130 / 3.7+5.74 /\left(\mathrm{B} 129^{\wedge} 0.9\right)\right)\right)^{\wedge}-2$ & 0.0165 & \\
\hline 132 & Friction Factor & $-0.25^{\circ}\left(\operatorname{LOG}\left(\mathrm{B} 130 / 3.7+2.51 /\left(\mathrm{B} 129^{\circ} \mathrm{B} 131^{\wedge} 0.5\right)\right)\right)^{\wedge}-2$ & 0.0164 & \\
\hline 133 & Pressure Drop & $=0.00000336^{*} \mathrm{~B} 132^{*} \mathrm{~B} 115^{*}\left(\mathrm{~B} 128^{\wedge} 2\right) / \mathrm{B} 120 /\left(\mathrm{B} 114^{\wedge} 5\right)$ & 9.705 & |psi \\
\hline 134 & & & & \\
\hline
\end{tabular}




\begin{tabular}{|c|c|c|c|c|}
\hline & $A$ & B & $\mathbf{C}$ & $\overline{\mathbf{D}}$ \\
\hline 135 & $\Delta P$ from Platform Bayonet to Outside & & & Units \\
\hline 136 & $>$ Inner Pipe Diameter & 0.53 & 0.53 & it \\
\hline 137 & Inner Pipe Diameter (conv.) & $=B 136 * 12$ & 6.36 & $\operatorname{in}$ \\
\hline 138 & $>$ Equivalent Length & 516 & 516 & $f t$ \\
\hline 139 & gAr Gas Density @ 1.5 bar \& Now Temp. & $=\mathrm{B} 90$ & 3.396 & $\mathrm{mg} / \mathrm{cc}$ \\
\hline 140 & gAr Gas Density @ 1.5 bar (comv.) & $=B 139 / 1000 * 62.428$ & 0.212 & $\mathrm{Ibm} / \mathrm{ft} \wedge 3$ \\
\hline 141 & gN2 Gas Density @ 1.5 bar \& New Temp. & $-B 118$ & 2.411 & $\mathrm{mg} / \mathrm{cc}$ \\
\hline 142 & gN2 Gas Density @ 1.5 bar (conv.) & $=B 141 / 1000 * 62.428$ & 0.151 & $16 \mathrm{~m} / \mathrm{ft}^{\wedge} 3$ \\
\hline 143 & Gas Mixture Density @1.5 bar & $=\left(\mathrm{B} 17^{*} \mathrm{~B} 140+\mathrm{B} 189^{*} \mathrm{~B} 142\right) / \mathrm{B} 149$ & 0.189 & $16 \mathrm{~m} / \mathrm{ft}^{\wedge} 3$ \\
\hline 144 & gAr Viscosity @ 1.5 bar \& Now Temp. & $=B 121$ & 0.0001696 & $\mathrm{~g} / \mathrm{cm} \cdot \mathrm{s}$ \\
\hline 145 & gAr Viscosity @ 1.5 bar (conv.) & $=B 144 * 100$ & 0.01696 & centipois $\theta$ \\
\hline 146 & gN2 Viscosity @ 1.5 bar \& Now Temp. & $=B 123$ & 0.0001365 & $\mathrm{~g} / \mathrm{cm}-\mathrm{s}$ \\
\hline 147 & gN2 Viscosity @ 1.5 bar (conv.) & $=B 146 * 100$ & 0.01365 & centipoise \\
\hline 148 & Gas Mixture Viscosity @1.5 bar & $=\left(\mathrm{B} 17^{*} \mathrm{~B} 145+\mathrm{B} 189^{*} \mathrm{~B} 147\right) / \mathrm{B} 149$ & 0.01573 & centipoise \\
\hline 140 & Max. Mass Flow of Gas Mixture & $=B 128$ & 13093 & $\mathrm{lbm} / \mathrm{hr}$ \\
\hline 150 & Reynolds Number & $=6.31^{\circ} \mathrm{B} 149 /\left(\mathrm{B} 137^{\circ} \mathrm{B} 148\right)$ & 826000 & \\
\hline 151 & > Relative Roughness (e/D) & 0.00027 & 0.00027 & \\
\hline 152 & Friction Factor Guess & $=0.25^{*}\left(\operatorname{LOG}\left(\mathrm{B} 151 / 3.7+5.74 /\left(\mathrm{B} 150^{\wedge} 0.9\right)\right)\right)^{\wedge}-2$ & 0.0156 & \\
\hline 153 & Friction Factor & $=0.25^{*}\left(\operatorname{LOG}\left(B 151 / 3.7+2.51 /\left(B 150^{*} B 152^{\wedge} 0.5\right)\right)\right)^{\wedge}-2$ & 0.0155 & \\
\hline 154 & Pressure Drop & $=0.00000336^{*} B 153^{\circ} B 138^{*}\left(B 149^{\wedge} 2\right) / B 143 /\left(B 137^{\wedge} 5\right)$ & 2.345 & psi \\
\hline 155 & & & & \\
\hline 156 & Summation of Equivalent $\Delta \mathrm{Ps}$ & & & Units \\
\hline 157 & Relief Valve Inlet Pressure Drop & $=\mathrm{B} 33$ & 1.655 & psi \\
\hline 158 & Rellef Valve Outlet Pressure Drop & $=B 65$ & 0.642 & psi \\
\hline 150 & Rellef Valve Pressure Drop & $=B 110$ & 5.403 & psi \\
\hline 160 & Relief Valve/Disk Branch & $=\mathrm{B} 33+\mathrm{B} 65+\mathrm{B} 110$ & 7.7 & psi \\
\hline 161 & Rupture Disk Inlet Pressure Drop & $=\mathrm{B} 48$ & 6.394 & psi \\
\hline 162 & Rupture Disk Outlet Prossure Drop & $=\mathrm{B} 80$ & 0.219 & psi \\
\hline 163 & Rupture Disk Pressure Drop & $=\mathrm{B} 157+\mathrm{B} 158+\mathrm{B} 159-\mathrm{B} 161-\mathrm{B} 162$ & 1.087 & psi \\
\hline 164 & Common Outlet Pressure Drop & $=B 133$ & 9.705 & psi \\
\hline 165 & Platform to Outside Pressure Drop & $=B 154$ & 2.345 & psi \\
\hline 166 & & & & \\
\hline
\end{tabular}




\begin{tabular}{|c|c|c|c|c|}
\hline & $\overline{\mathbf{A}}$ & $\mathbf{B}$ & $\mathbf{C}$ & D \\
\hline 167 & $\Delta \mathrm{P}$ Across Rupture Disk & & & Units \\
\hline 168 & $>$ Argon Specific Heat Ratio (k) & 1.673 & 1.673 & \\
\hline 169 & Critical Ratio & $=(2 /(B 168+1))^{\wedge}(B 168 /(B 168-1))$ & 0.486 & \\
\hline 170 & $>$ Area of 3" Rupture Disk & $-3.14159^{\star}\left(3^{\wedge} 2\right) / 4$ & 7.069 & $\operatorname{in} 2$ \\
\hline 171 & Flow Through Rupture Disk & $=\mathrm{B} 43$ & 4281 & $\mathrm{lb} \mathrm{m} / \mathrm{hr}$ \\
\hline 172 & Flowing Temperature & $-1.8^{*} \mathrm{B2}$ & 522 & $\operatorname{deg} R$ \\
\hline 173 & $>$ ASME Coefficient (K) & 0.62 & 0.62 & \\
\hline 174 & Pressure Ratio (Pe/Po) & $=\mathrm{B} 179 / \mathrm{B} 176$ & 0.961 & \\
\hline 175 & gAr Flow Constant for Subsonic Flow(C1) & $=\operatorname{SQRT}\left(2^{*} 32.2 / 1545^{\circ}(\mathrm{B} 168 /(\mathrm{B} 168-1))^{*}\left(\mathrm{~B} 174^{\wedge}(2 / \mathrm{B} 168)-\mathrm{B} 17\right.\right.$ & 0.039 & \\
\hline 176 & Flowing Inlet Pressure (Po) & $-B 4+14.696-B 48$ & 28.05 & psia \\
\hline 177 & > Molecular Weight of Argon & 39.948 & 39.948 & g/mol \\
\hline 178 & Critical Pressure (PCr) & $=B 169^{*} \mathrm{~B} 176$ & 13.64 & psia \\
\hline 179 & Outlet Pressure ( $\mathrm{P \theta}$ ) (using delta $\mathrm{p}^{\prime} \mathrm{s}$ ) & $=14.696+B 154+B 133+B 80$ & 26.97 & psia \\
\hline 180 & Pressure Drop Across Rupture Disk & $=\mathrm{B} 176-\mathrm{B} 179$ & 1.087 & psi \\
\hline 181 & Maximum Theoretical Rupture Disk Flow & $-\mathrm{B} 170^{*} \mathrm{~B} 173^{*} \mathrm{~B} 175^{*} \mathrm{~B} 176^{*} \mathrm{SORT}(\mathrm{B} 177 / \mathrm{B} 172)^{*} 60^{*} 60$ & 4833 & $\mathrm{ibm} / \mathrm{hr}$ \\
\hline 182 & Actual Rupture Disk Flow & $=\mathrm{B} 43$ & 4281 & $\mathrm{lb} \mathrm{m} / \mathrm{hr}$ \\
\hline \multicolumn{5}{|c|}{\begin{tabular}{|l|l|}
183 & -2 \\
\end{tabular}} \\
\hline 184 & Maximum Flow from Condensers & & & units \\
\hline 185 & $>$ Max. Flow of Liquid Nitrogen & 13 & 13 & gpm \\
\hline 186 & Max. Flow of Liquid Nitrogen (conv.) & $=8185 / 7.48$ & 1.74 & $f^{\wedge} 3 / \mathrm{min}$ \\
\hline 187 & Density of LN2@ $3.5 \mathrm{~atm}$ & 0.747 & 0.747 & $\mathrm{~g} / \mathrm{cc}$ \\
\hline 188 & Density of LN2 (conv.) & $=B 187^{*} 62.4$ & 46.6128 & $1 \mathrm{bm} / \mathrm{ft}^{\wedge} 3$ \\
\hline 189 & Mass Flow of LN2 & $=B 186^{*} B 188^{*} 60$ & 4861 & $\mathrm{lbm} / \mathrm{hr}$ \\
\hline
\end{tabular}




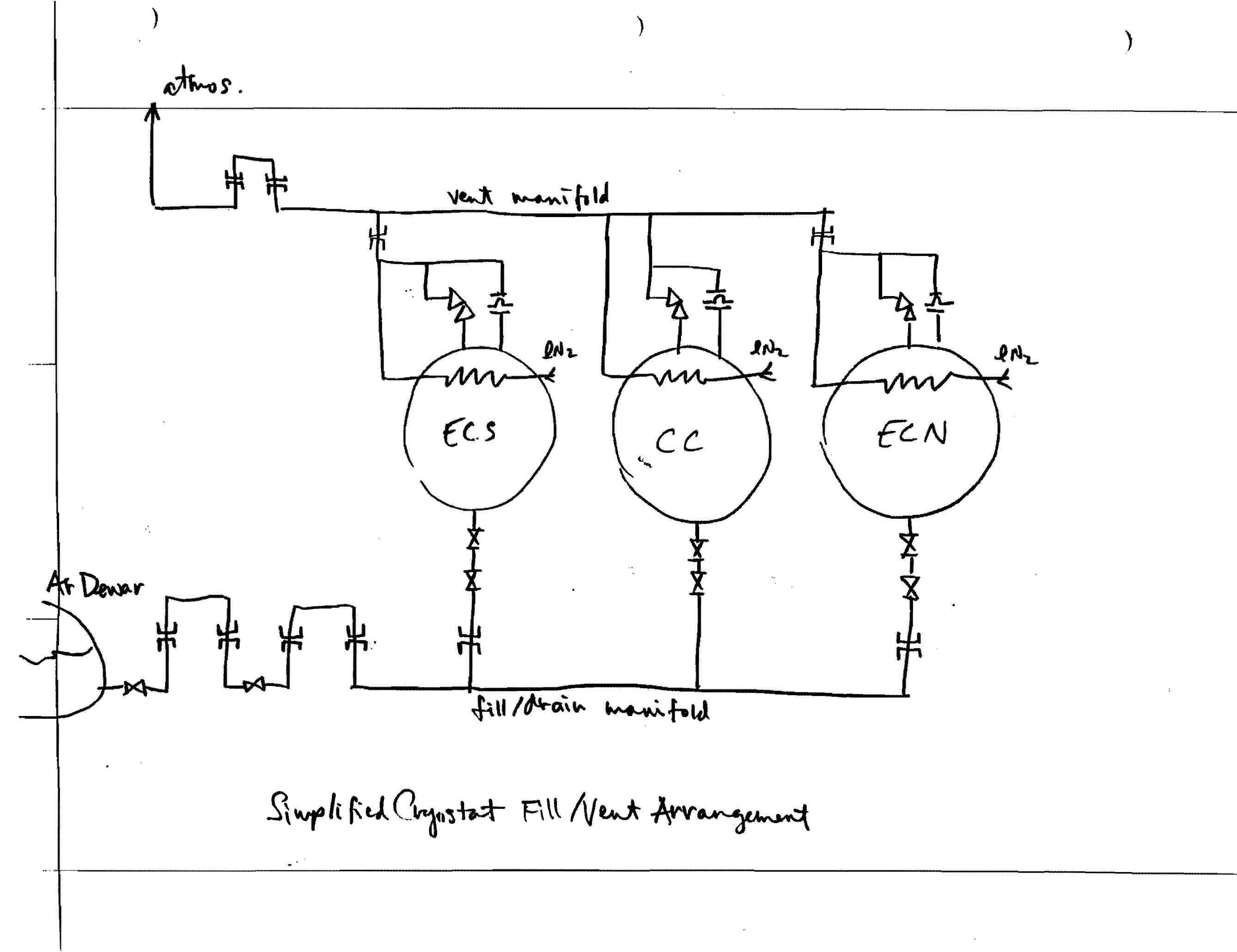




\section{Calculation of Max. Flowrate from LAr Dewar to ECN}

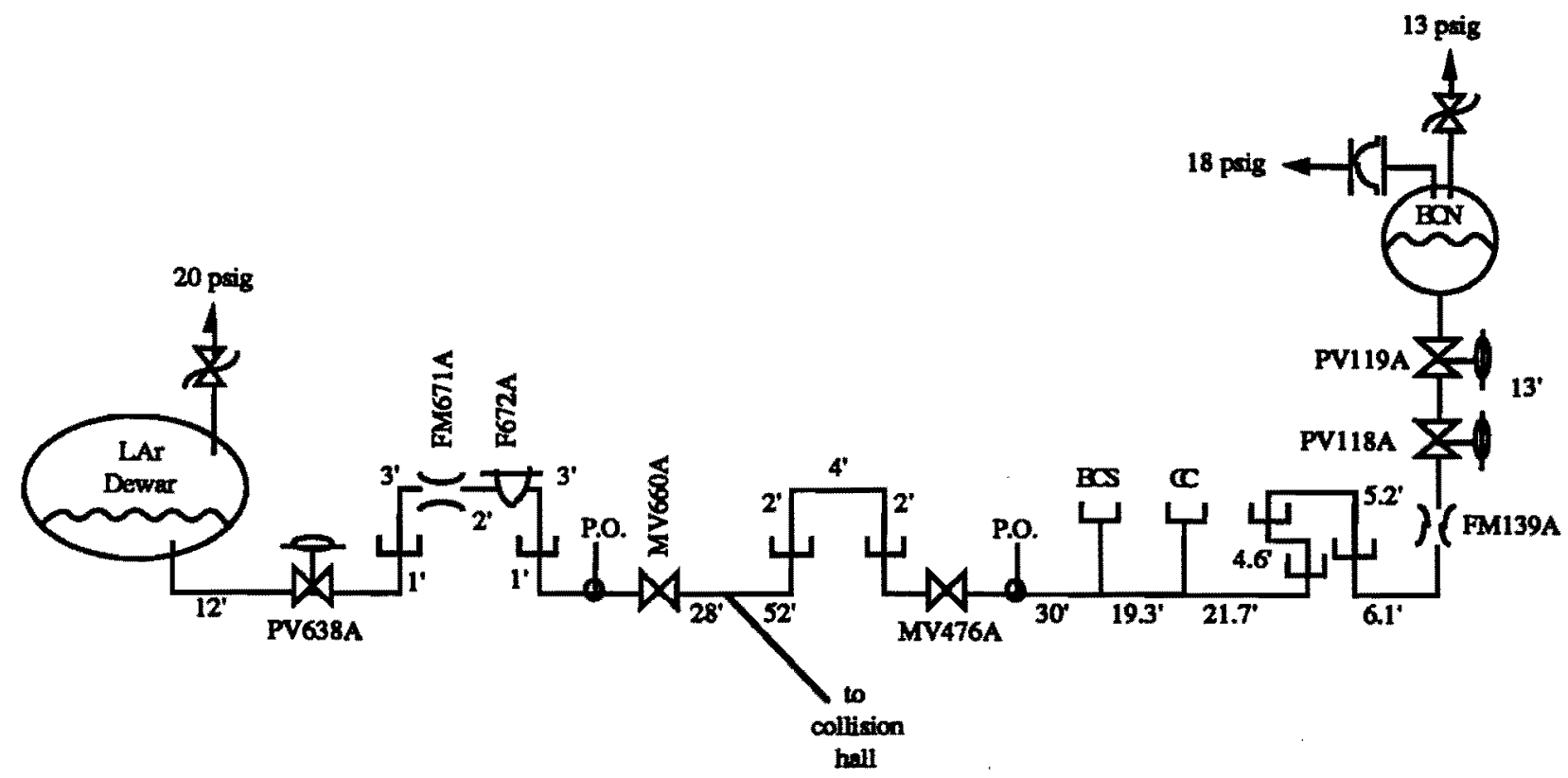

$\mathrm{d}=1.682 \mathrm{in}=0.1402 \mathrm{ft}$

[1 1/2" SCH. 10 inner pipe dia.]

$\mathrm{A}=0.01543 \mathrm{ft}^{2}$

[cross-sectional area]

\begin{tabular}{|l|c|c|c|c|}
\hline $\begin{array}{l}\text { Reference: drawings } \\
\text { from Tony Parker }\end{array}$ & up to cryocomer & cryocomer to CC & CC to ECN & TOTAL \\
\hline \# of elbows, 90 & 17 & 4 & 19 & 40 \\
\hline \# of elbows, 45 & 1 & 3 & 1 & 5 \\
\hline \# of tees, branch & 3 & 0 & 0 & 3 \\
\hline \# of tees, thru & 0 & 2 & 0 & 2 \\
\hline
\end{tabular}




\section{Calculation of Equivalent Length}

Calculate the equivalent length of the piping from the LAr dewar to the inner vessel of the ECN.

$\mathrm{L}_{\text {piping }}=\mathrm{L}_{1.7}$ "dia. $+\mathrm{L}_{1.0 \text { "dia. }}$

Adjust $169 \mathrm{ft}$ length to CC (ref. Kelly Dixon) to include ECN.

$\mathrm{L}_{1.7 \text { " dia. }}=169 \mathrm{ft}$ (length to $\left.\mathrm{CC}\right)-8.3 \mathrm{ft}(\mathrm{CC}$ drain line) $+21.7 \mathrm{ft}$ (CC to rotary bayonet assembly) $+4.6 \mathrm{ft}+5.2 \mathrm{ft}$ (rotary U-tube dimensions) +6.1

$\mathrm{ft}+13 \mathrm{ft}(\mathrm{ECN}$ drain line $)=211.3 \mathrm{ft}$ total.

Equivalent lengths of the flowmeters are accounted for by including a 4 foot length of 1.0" diameter piping. Convert 1.0" diameter equivalent length to 1.7 " dia. equivalent length:

Reference: Crane Technical Paper No. 410

(1" SCH. 40 to $11 / 2$ " SCH. 10)

$L_{1.0^{\prime \prime} \text { dia. }}=\left(\frac{1.682}{1.049}\right)^{5} \times 4^{\prime}=42.4^{\prime}$

$\mathrm{L}_{\text {piping }}=211.3 \mathrm{ft}+42.4 \mathrm{ft}=253.7 \mathrm{ft}$ total

Convert elbows and tees into equivalent lengths of pipe.

$\mathrm{L}_{\text {fittings }}=[40(20)+5(14)+3(60)+2(20)] \times 0.1402 \mathrm{ft}=152.8 \mathrm{ft}$

$\mathrm{L}_{\text {eq }}=253.7 \mathrm{ft}+152.8 \mathrm{ft}=406.5 \mathrm{ft}$ 


\section{Calculation of Resistance Coefficient}

Reference: Crane Technical Paper No. 410

Calculate the resistance coefficient for the piping and fittings.

$K_{\text {piping, fittings }}=f\left(\frac{L_{e q}}{d}\right)$

let $\mathrm{f}=\mathbf{0 . 0 2 2}$

[friction factor guess]

$\mathrm{K}_{\text {piping, fittings }}=0.022\left(\frac{406.5 \mathrm{ft}}{0.1402 \mathrm{ft}}\right)=63.79$

Include inlet and outlet losses (ref. Kelly Dixon).

$K_{\text {inlet }}=0.5$

$\mathrm{K}_{\text {outlet }}=1.0$

Calculate resistance coefficient for the valves.

$K_{\text {valves }}=\left(\frac{29.9 \mathrm{~d}^{2}}{C_{v}}\right)^{2} \times(\#$ of valves $)=\left(\frac{29.9(1.682)^{2}}{34}\right)^{2} \times 4=24.76$

where the diameter, $d$, is in inches, not feet.

$\Sigma \mathrm{K}=63.79+1.5+24.76=90.05$ 


\section{Driving Pressure}

Calculate the differential pressure available under relieving conditions.

max. head available = 720.3 ft (dewar@ 16,000 gallons)

- $715.2 \mathrm{ft}$ (bottom of ECN)

$5.1 \mathrm{ft}$ (total elevation difference)

Calculate the pressure due to elevation difference.

Density of liquid argon @ 19.75 psig=1.337 g/cc, which corresponds to a specific weight of $0.580 \mathrm{psi} / \mathrm{ft}$.

$\Delta \mathrm{p}$ due to head $=5.1 \mathrm{ft} \times 0.580 \mathrm{psi} / \mathrm{ft}=2.96 \mathrm{psi}$

$\Delta \mathrm{p}_{\text {relieving }}=($ LAr dewar pressure $)-($ ECN pressure $)+($ head pressure $)$

$$
=34.7 \text { psia }-34.45 \text { psia }+2.96 \text { psi }=3.21 \text { psid }
$$

Determine the pressure drop across the cryofilter.

Actual experience shows that with a $30 \mathrm{gpm}$ flow, the pressure drop across the cryofilter is 4 pṣid.

$\Delta \mathrm{p}_{\text {filter }}=\left(\frac{\mathrm{q}}{30}\right)^{2} \times 4 \mathrm{psid}=0.00444 \mathrm{q}^{2}$

where $\Delta p_{\text {filter }}$ is in psid if $q$ is in $g p m$.

$\Delta \mathrm{p}_{\text {available }}=\Delta \mathrm{p}_{\text {relieving }}-\Delta \mathrm{p}_{\text {filter }}$ 


\section{Calculation of Flowrate}

Calculate the flowrate, q, by rearranging Darcy's formula (ref. Crane 410). modified Darcy's formula:

$\Delta \mathrm{p}=\frac{\rho \Sigma \mathrm{K}}{144} \frac{\mathrm{v}^{2}}{2 \mathrm{~g}_{\mathrm{c}}}$

where: $\Delta \mathrm{p}$ is in psid,

$\rho$ is in $l b_{m} / f^{3}$,

and $v$ is in $f t / s$.

(144 is a conversion factor of $\mathrm{in}^{2} / \mathrm{ft}^{2}$.)

Rearrange to solve for the velocity, $v$.

$$
\begin{aligned}
& v=\sqrt{\frac{2 \mathrm{~g}_{\mathrm{c}}(144 \Delta \mathrm{p})}{\rho \Sigma K}}=\sqrt{\frac{2\left(32.174 \frac{1 \mathrm{~b}_{\mathrm{m}} \mathrm{ft}}{1 \mathrm{ft}_{\mathrm{f}} \mathrm{s}^{2}}\right)\left(144 \frac{\mathrm{in}^{2}}{\mathrm{ft}^{2}}\right) \Delta \mathrm{p}_{\text {available }}}{\left(83.47 \frac{1 \mathrm{~b}_{\mathrm{m}}}{\mathrm{ft}^{3}}\right)(90.05)}} \\
& \mathrm{v}=\begin{array}{c}
1.110 \sqrt{\Delta \mathrm{p}_{\text {available }}} \\
\text { where } \mathrm{v} \text { is in } \mathrm{ft} / \mathrm{s} \text { if } \Delta \mathrm{p}_{\text {available }} \text { is in psid. }
\end{array}
\end{aligned}
$$


Substitute formulas with $q$ into both sides of the equation for $v$ and $\Delta p$.

Substitute for $\mathrm{v}$ : (Let $\mathrm{Q}$ be the flow rate in $\mathrm{cfs}$.)

$$
\begin{gathered}
v=\frac{Q}{A}=\frac{4 Q}{\pi d^{2}}=\frac{4 Q}{\pi(0.1402 \mathrm{ft})^{2}}=64.78 Q \\
\text { where } v \text { is in } \mathrm{ft} / \mathrm{s} \text { if } \mathrm{Q} \text { is in cfs. }
\end{gathered}
$$

Convert the equation so that $\mathrm{v}$ will be in $\mathrm{ft} / \mathrm{s}$ if $\mathrm{q}$ is in $\mathrm{gpm}$.

$v=64.78\left(q \frac{\mathrm{gal}}{\mathrm{min}} \times \frac{\mathrm{min}}{60 \mathrm{sec}} \times 0.13368 \frac{\mathrm{ft}^{3}}{\mathrm{gal}}\right)=0.1443 \mathrm{q}$ where $v$ is in $\mathrm{ft} / \mathrm{s}$ if $\mathrm{q}$ is in $\mathrm{gpm}$.

Substitute for $\Delta \mathrm{p}$ :

From before, $\Delta \mathrm{p}_{\text {available }}=\Delta \mathrm{p}_{\text {relieving }}-\Delta \mathrm{p}_{\text {filter. }}$

$$
\Delta \mathrm{p}_{\text {available }}=3.21 \mathrm{psid}-0.00444 \mathrm{q}^{2}
$$

where $\Delta p_{\text {available }}$ is in psid if $q$ is in $\mathrm{gpm}$.

From before,

$$
\mathrm{v}=1.110 \sqrt{\Delta \mathrm{p}_{\text {available }}}
$$

where $v$ is in $f t / s$ if $\Delta p_{\text {available }}$ is in psid.

Substitute formula for $\Delta \mathrm{p}_{\text {available }}$ to get $\mathrm{v}$ in terms of $\mathrm{q}$.

$$
\begin{aligned}
& v=1.110 \sqrt{3.21-0.00444 q^{2}} \\
& \text { where } v \text { is in } \mathrm{ft} / \mathrm{s} \text { if } \mathrm{q} \text { is in } \mathrm{gpm} \text {. }
\end{aligned}
$$


Set the two equations for $v$ in terms of $q$ equal, and solve for $q$.

$v=0.1443 q=1.110 \sqrt{3.21-0.00444 q^{2}}$

$q=\sqrt{\frac{3.21}{\left(\frac{0.1443}{1.110}\right)^{2}+0.00444}}$

$\mathrm{q}=12.26 \mathrm{gpm}$

\section{Check_Friction Factor}

$\operatorname{Re}_{\mathrm{d}_{\text {relieving }}}=\frac{\rho \mathrm{V}_{\mathrm{re1}} \mathrm{d}}{\mu}$

$\mu=2.4185 \times 10^{-3} \frac{\mathrm{g}}{\mathrm{cm}-\mathrm{s}} @$ sat. $1.3 \mathrm{bars}=1.6252 \times 10^{-4} \frac{1 \mathrm{~b}_{\mathrm{m}}}{\mathrm{ft}-\mathrm{s}}$

$\mathrm{q}_{\text {relieving }}=12.26 \mathrm{gpm}$

$\mathrm{v}_{\mathrm{rel} .}=\frac{\mathrm{q}_{\text {relieving }}}{\mathrm{A}}=\frac{4 \mathrm{q}_{\text {relieving }}}{\pi^{2}}=\frac{4\left(12.26 \mathrm{gpm} \times \frac{1 \mathrm{cfs}}{448.83 \mathrm{gpm}}\right)}{\pi(0.1402 \mathrm{ft})^{2}}=1.769 \frac{\mathrm{ft}}{\mathrm{s}}$

$\operatorname{Re}_{\mathrm{d}_{\text {relieving }}}=\frac{\left(83.47 \frac{1 \mathrm{~b}_{\mathrm{m}}}{\mathrm{ft}^{3}}\right)\left(1.769 \frac{\mathrm{ft}}{\mathrm{s}}\right)(0.1402 \mathrm{ft})}{1.6252 \times 10^{-4} \frac{1 \mathrm{~b}_{\mathrm{m}}}{\mathrm{ft}-\mathrm{s}}}=1.274 \times 10^{5}$

Assuming a value of 0.00015 roughness for commercial steel pipe, the relative roughness is 0.001 , and the friction factor is 0.022 , which checks. 


\section{TK Solver Plus Analysis}

The following two pages are printouts of the variable and rule sheet from a TK Solver model set up to verify the hand calculations for the maximum flow rate to the ECN from the Argon dewar. The first page is the variable sheet, which shows the typed inputs and calculated outputs for various parameters. The complete solution requires guessing a number for the friction factor. The program then iterates to find the exact solution. Also note that the columns have a set width, so that not all of the entries are shown completely, specifically, $g$ has units of $1 \mathrm{bm}-\mathrm{ft} / \mathrm{lbf}-\mathrm{s}^{\wedge} 2$. The second sheet is the rule sheet, which shows the various formulas used. The complete model has been saved in the Co-op Mac, under the name "TK LAD Flow to ECN". 


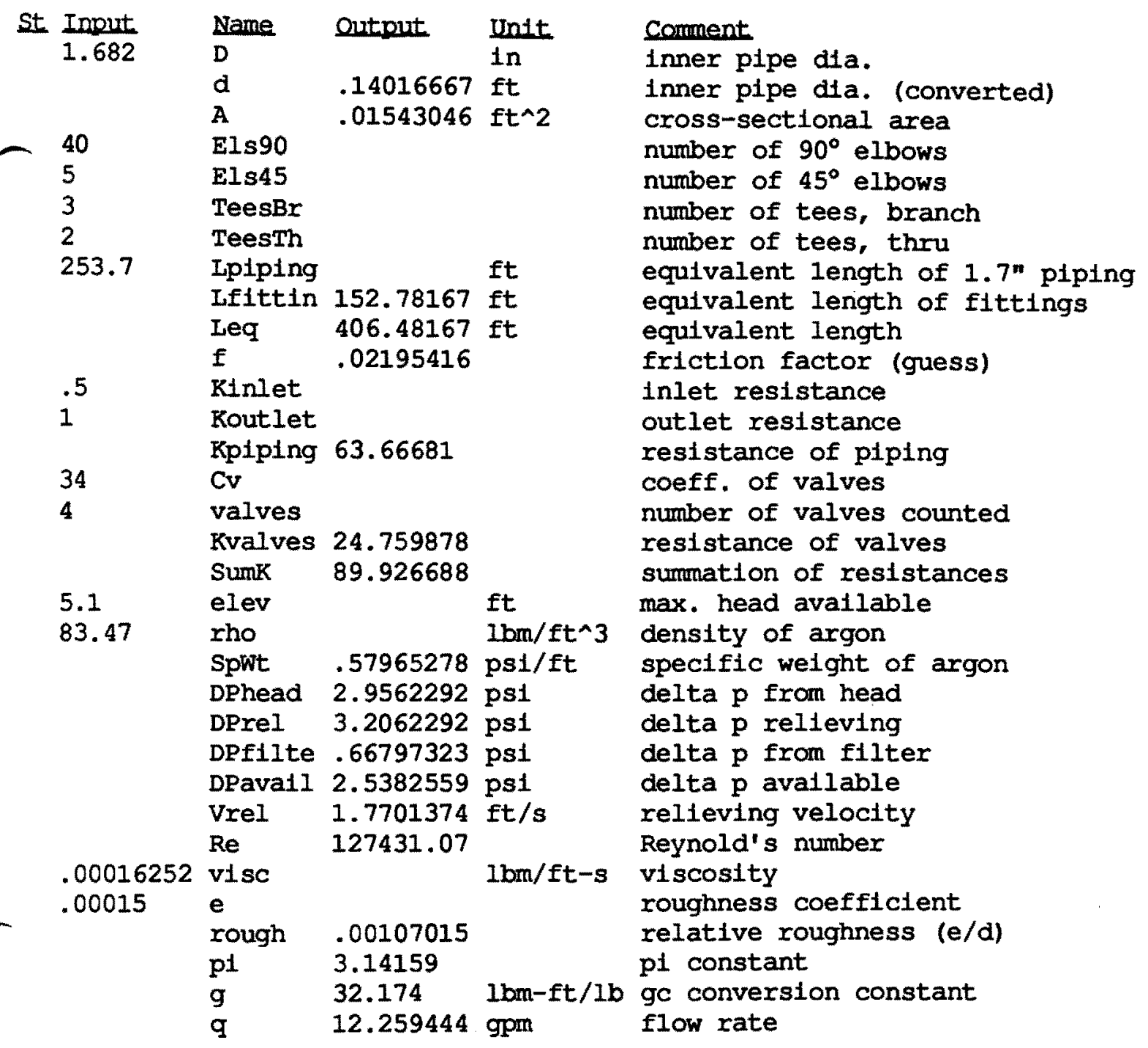


S Rule

* $\mathrm{p} 1=3.14159$

$\star \mathrm{g}=32.174$

$\star \mathrm{d}=\mathrm{D} / 12$

"constant for $\mathrm{p} 1$

"constant for gc, in units of (1bm-ft)/(1bf-s^2)

$-A=p 1^{*}\left(d^{\wedge} 2\right) / 4$ "conversion of inner pipe dia. from inches to feet Ifittings $=d^{*}($ Els $90 * 20+E 1 s 45 * 14+$ TeesBr*60+TeesTh*20)

"equivalent length

* Leq $=$ Ipiping + Ifittings

"total equivalent length of $1.7^{\text {" dia piping }}$

* Kpiping $=f * I e q / d$ "resistance coeff. for piping and fittings

* Kvalves $=$ valves* $\left(\left(29.9 *\left(D^{\wedge} 2\right) / C v\right)^{\wedge} 2\right)$ "resistance of valves

* Sumk = Kpiping+Kvalves+Kinlet +Koutlet "summation of $\mathrm{K}$ coeff.

* SpWt $=$ rho/144

* DPhead $=$ elev $\star$ SpWt

* DPrel $=34.7-34.45+$ DPhead

* DPfilter $=4 *\left((q / 30)^{\wedge} 2\right)$

* DPavail $=$ DPrel - DPfilter

* DPavail $=($ rho* SunK* $($ Vrel^2)

* Vrel $=\left(q^{\star} .13368 / 60\right) / A$

* $\operatorname{Re}=r h o * V r e l * d / v i s c$

"summation density

$\star$ rough $=e / d$

"relative roughness for commercial steel

$\star 1 /\left(f^{\wedge} .5\right)=-2.0 * \log \left((\right.$ rough $\left./ 3.7)+\left(2.51 /\left(\operatorname{Re}^{\star}\left(f^{\wedge} .5\right)\right)\right)\right)$ "Moody chart

due to max. head pressure

"delta $p$ due to elevation difference

"pressure drop across cryofilter

"available differential driving pressure

)$/(144 * 2 * g)$

"modified Darcy's formula

"velocity in $\mathrm{ft} / \mathrm{s}$ from flow rate in gpm

"Reynold's number for pipe flow 


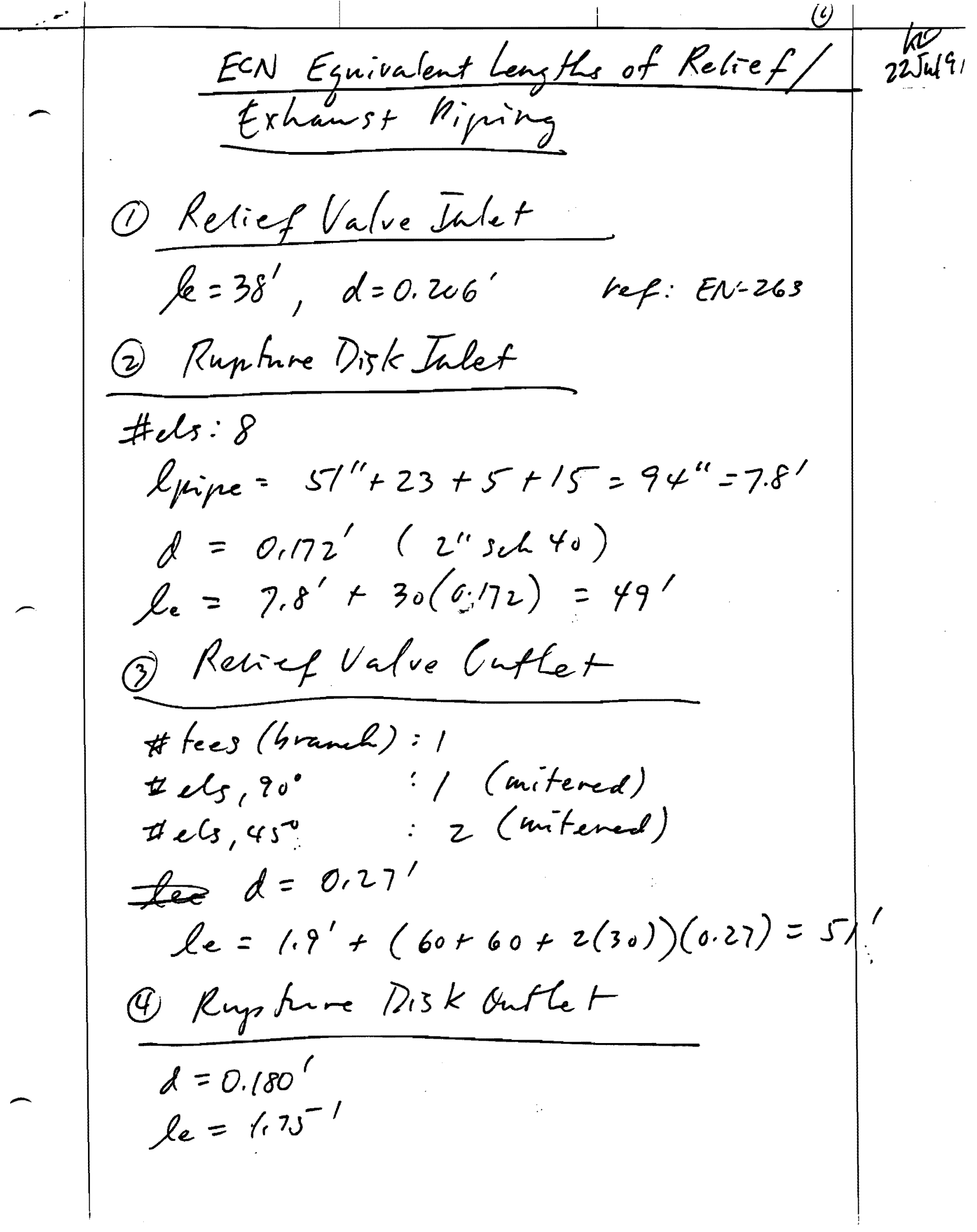




\section{Determine Worst Case Cryostat to Fill Due to Pressure}

\section{Fill capacity}

Reference: ECN numbers taken from previous "Calculation of Max. Flowrate from LAr Dewar to ECN".

$$
\begin{aligned}
& \left(\mathrm{q}_{\mathrm{fiH}}^{\mathrm{ECN}}\right)_{\max }=12.3 \mathrm{gpm} \\
& \Sigma K^{\mathrm{ECN}}=90.05 \\
& \Sigma K^{\propto}=\Sigma K^{\mathrm{ECN}}-\frac{\mathrm{f}\left(\mathrm{L}_{\mathrm{eq}}\right)}{\mathrm{d}} \cong 90.05-\frac{(0.022)(30 \mathrm{ft})}{0.1402 \mathrm{ft}}=85.34 \\
& \Sigma K^{\mathrm{ECS}}=\Sigma K^{\propto C}-\frac{f\left(\mathrm{~L}_{\mathrm{eq}}\right)}{\mathrm{d}} \cong 85.34-\frac{(0.022)(27 \mathrm{ft})}{0.1402 \mathrm{ft}}=81.10
\end{aligned}
$$

The ECS has the smallest resistance coefficient because it has the shortest equivalent length of piping from the argon dewar. Therefore, the same driving pressure from the dewar will produce the largest inlet flow to the ECS. The flowrate is inversely proportional to the square root of the resistance coefficient:

$$
q^{\text {cryostat }} \propto \frac{1}{\sqrt{\Sigma K^{\text {cryostat }}}}
$$

Therefore, the flowrates of the ECS and ECN can be compared as follows:

$$
\frac{\mathrm{q}_{\mathrm{fill}}^{\mathrm{ECS}}}{\mathrm{q}_{\mathrm{fi}}^{\mathrm{ECN}}}=\frac{\sqrt{\Sigma K^{\mathrm{ECN}}}}{\sqrt{\Sigma K^{E C S}}}=\frac{\sqrt{90.05}}{\sqrt{81.10}}=1.054
$$


The maximum inlet flow to the ECS is greater than the maximum inlet flow to the ECN by about 5\%. However, the ECS is not the worst case cryostat to fill because it also has a shorter equivalent length of relief piping.

\section{Venting capacity}

Reference: D $\emptyset$ Engineering Note 3740.224-EN-323, ECN Pressure and Vacuum Vessel Engineering Notes.

The first spreadsheet in EN-323 calculates the ECN relief flow capacity. In the section, " $\Delta \mathrm{P}$ Across Relief Valve", line 108 shows the maximum theoretical flow through the relief valve. In the section, " $\Delta \mathrm{P}$ Across Rupture Disk", line 181 shows the maximum theoretical flow through the rupture disk. Therefore, the total mass flow can be calculated as:

$\rho\left(\mathrm{q}_{\mathrm{rel}}^{\mathrm{ECN}}\right)_{\max }=7740 \frac{1 \mathrm{~b}_{\mathrm{m}}}{\mathrm{hr}}($ relief valve $)+7458 \frac{1 \mathrm{~b}_{\mathrm{m}}}{\mathrm{h} \mathrm{r}}($ rupture disk $)=15,198 \frac{1 \mathrm{~b}_{\mathrm{m}}}{\mathrm{h} \mathrm{r}}$

Note that $q$ here represents the flow through the relief piping, out of the $E C N$, whereas the $q$ on the previous page represented the inlet fill piping, into the ECN.

The calculation of the venting capacity of the ECS is more complicated, because the numbers can not be referenced from EN-323. To calculate the ECS capacity, the ECN spreadsheet was modified for the ECS, changing the section, " $\Delta \mathrm{P}$ Across Common Outlet to Cryocorner". Line 115 is the equivalent length of this section of piping. ECN had an equivalent length of $273 \mathrm{ft}$, which was calculated in this note (see previous K. Dixon's hand calculations, "ECN Equivalent Lengths of Relief/Exhaust Piping"). The only difference in the relief piping of the ECS is that the equivalent length of the common outlet changes to $238 \mathrm{ft}$. Using this new equivalent length, the spreadsheet was re-calculated to find the maximum theoretical flows through the relief valve and rupture disk. The following pages show the actual spreadsheet, modified for the ECS. 


\begin{tabular}{|c|c|c|c|c|}
\hline & A & B & C & D \\
\hline 1 & Conversion of Liquid to Gas at Module Temp. & & & Units \\
\hline 2 & $>$ Bulk Temp. of Modules & 96 & 96 & $\mathrm{~K}$ \\
\hline 3 & >* Max. Liquid Equivalent Flow of Argon to Reliefs & 24.9 & 24.9 & gpm \\
\hline 4 & $>$ Pressure in Cryostat & 19.75 & 19.75 & psig \\
\hline 5 & Pressure in Cryostat (conv.) & $=(B 4 / 14.696+1) * 1.01325$ & 2.375 & bars \\
\hline 6 & $>\operatorname{lAr}$ Density@ 2.2 bars & 1.342421 & 1.342 & $g / c c$ \\
\hline 7 & $>$ IAr Density @ 2.4 bars & 1.335861 & 1.336 & $g / c c$ \\
\hline 8 & Ar Density @ 2.375 bars & $=(B 5-2.2) / 0.2^{*}(B 7-B 6)+B 6$ & 1.337 & $g / c c$ \\
\hline 9 & $>$ gAr Density @ 2.2 bars & 11.77 & 11.77 & $\mathrm{mg} / \mathrm{cc}$ \\
\hline 10 & $>$ gAr Donsity @ 2.4 bars & 12.75 & 12.75 & mg/cc \\
\hline 11 & gAr Density @ 2.375 \& Temp. & $=(\mathrm{B} 5-2.2) / 0.2^{*}(\mathrm{~B} 10-\mathrm{B9})+\mathrm{B9}$ & 12.627 & $\mathrm{mg} / \mathrm{cc}$ \\
\hline 12 & gAr Density @ 2.375 bars (conv.) & $=B 11 / 1000 * 62.428$ & 0.788 & $1 \mathrm{bm} / \mathrm{ft}^{\wedge} 3$ \\
\hline 13 & Maximum Flow of Argon Gas & $=B 3^{*} \mathrm{~B} 8 / \mathrm{B} 11^{*} 1000$ & 2636 & gpm \\
\hline 14 & Maximum Flow of Argon Gas (conv.) & $=B 13^{*} 0.13368$ & 352 & $\mathrm{cfm}$ \\
\hline 15 & Air Equivalent Flow @STP & $=6.32^{*} \mathrm{~B} 17^{*} 356 / \mathrm{B} 16^{*} \mathrm{SQRT}\left(\mathrm{B} 99 /\left(520^{*} \mathrm{~B} 177^{*} 28.97\right)\right)$ & 1658 & scfm air \\
\hline 16 & Specific Heat Constant, C, for Ar & $=520^{*} \mathrm{SQRT}\left(\mathrm{B} 168^{*}(2 /(\mathrm{B} 168+1))^{\wedge}((\mathrm{B} 168+1) /(\mathrm{B} 168-1))\right)$ & 383 & \\
\hline 17 & Max. Mass Flow of Argon Gas & $=\mathrm{B} 14^{*} \mathrm{~B} 12^{*} 60$ & 16666 & $\mathrm{ibm} / \mathrm{hr}$ \\
\hline 18 & $>$ Percent of Mass Flow to Relief Valve & 0.474 & 0.474 & \\
\hline 19 & & & & \\
\hline 20 & $\Delta P$ Across Relief Valve Inlet & & & Units \\
\hline 21 & $>$ Inner Pipe Diameter & 0.206 & 0.206 & 11 \\
\hline 22 & Inner Pipe Diameter (conv.) & $=B 21 * 12$ & 2.472 & in \\
\hline 23 & $>$ Equivalent Length & 38 & 38 & $f t$ \\
\hline 24 & Ar Gas Density @ 2.375 \& Temp. & $=(B 5-2.2) / 0.2^{*}(\mathrm{~B} 10-\mathrm{B} 9)+\mathrm{B} 9$ & 12.627 & $\mathrm{mg} / \mathrm{cc}$ \\
\hline 25 & Ar Gas Density @ 2.375 bars (conv.) & $=B 24 / 1000^{*} 62.428$ & 0.788 & $1 \mathrm{bm} / \mathrm{ft}^{\wedge} 3$ \\
\hline 26 & $>$ gAr Viscosity @ 2.4 bars \& Tomp. & 0.0000803 & 0.0000803 & $\mathrm{~g} / \mathrm{cm}-\mathrm{s}$ \\
\hline 27 & gAr Viscosity @ 2.4 bars (conv.) & $=B 26 * 100$ & 0.00803 & centipoise \\
\hline 28 & Max. Mass Flow to Relief Valve & $=B 17^{*} B 18$ & 7900 & $\mathrm{lbm} / \mathrm{hr}$ \\
\hline 29 & Reynolds Number & $=6.31^{*} \mathrm{~B} 28 /\left(\mathrm{B} 22^{*} \mathrm{~B} 27\right)$ & 2510000 & \\
\hline 30 & > Relative Roughness (e/D) & 0.0007 & 0.0007 & \\
\hline 31 & Friction Factor Guess & $=0.25^{*}\left(\operatorname{LOG}\left(B 30 / 3.7+5.74 /\left(B 29^{\wedge} 0.9\right)\right)\right)^{\wedge}-2$ & 0.0183 & \\
\hline 32 & Friction Factor & $=0.25^{*}\left(\operatorname{LOG}\left(\mathrm{B} 30 / 3.7+2.51 /\left(\mathrm{B} 29^{*} \mathrm{~B} 31^{\wedge} 0.5\right)\right)\right)^{\wedge}-2$ & 0.0182 & \\
\hline 33 & Pressure Drop & $=0.00000336^{*} \mathrm{~B} 32^{*} \mathrm{~B} 23^{*}\left(B 28^{\wedge} 2\right) / B 25 /\left(B 22^{\wedge} 5\right)$ & 1.993 & psi \\
\hline 34 & & & & \\
\hline
\end{tabular}




\section{Maximum ECS Rellef Flow Calculation 10/18/91}

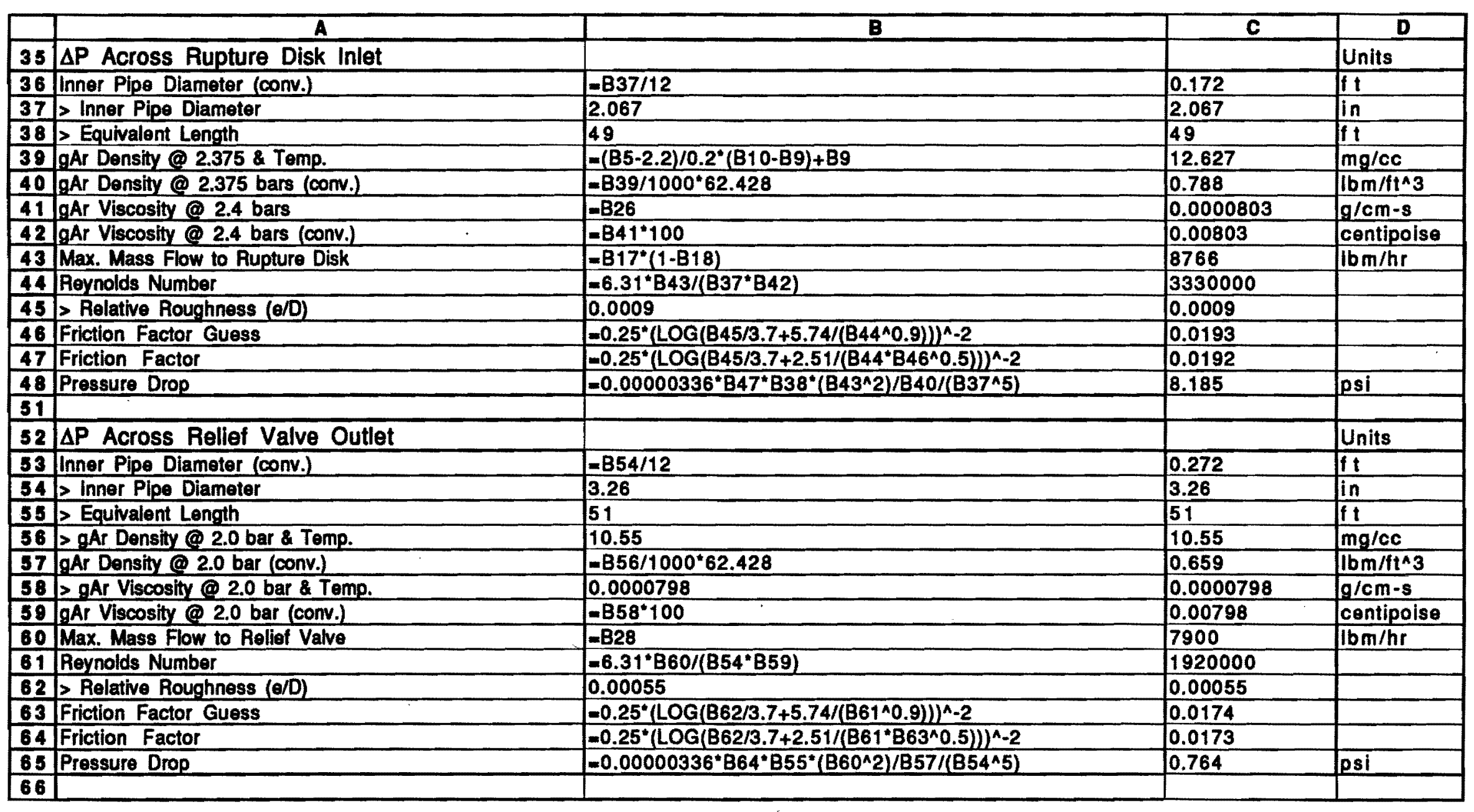




\begin{tabular}{|c|c|c|c|c|}
\hline & $\bar{A}$ & $\mathbf{B}$ & $\mathbf{C}$ & D \\
\hline 67 & $\Delta \mathrm{P}$ Across Rupture Disk Outlet & & & Units \\
\hline 68 & Inner Pipe Diameter (conv.) & $-869 / 12$ & 0.18 & $f t=$ \\
\hline 68 & > Inner Pipe Diameter & 2.157 & 2.157 & in \\
\hline 70 & $>$ Equivalent Length & 1.75 & 1.75 & ft \\
\hline 71 & gAr Density @ 2.0 bar \& Temp. & -B56 & 10.55 & $\mathrm{mg} / \mathrm{cc}$ \\
\hline 72 & gAr Density @ 2.0 bar (conv.) & $=B 71 / 1000^{*} 62.428$ & 0.659 & $1 \mathrm{bm} / \mathrm{ft}^{\wedge} 3$ \\
\hline 73 & gAr Viscosity @ 2.0 bar \& Temp. & $=\mathrm{B} 58$ & 0.0000798 & $\mathrm{~g} / \mathrm{cm}-\mathrm{s}$ \\
\hline 74 & gAr Viscosity @ 2.0 bar (conv.) & $=B 73 * 100$ & 0.00798 & centipoise \\
\hline 75 & Max. Mass Flow to Rupture Disk & -843 & 8766 & $\mathrm{lbm} / \mathrm{hr}$ \\
\hline 76 & Reynolds Number & $=6.31 * B 75 /(B 69 * B 74)$ & 3210000 & \\
\hline 77 & $>$ Relative Roughness $(\mathrm{e} / \mathrm{D})$ & 0.0009 & 0.0009 & \\
\hline 78 & Friction Factor Guess & $=0.25^{\circ}\left(\operatorname{LOG}\left(B 77 / 3.7+5.74 /\left(B 76^{\wedge} 0.9\right)\right)\right)^{\wedge}-2$ & 0.0193 & \\
\hline 79 & Friction Factor & $=0.25^{*}\left(L O G\left(B 77 / 3.7+2.51 /\left(B 76^{*} B 78^{n} 0.5\right)\right)\right)^{n}-2$ & 0.0192 & \\
\hline 80 & Pressure Drop & $=0.00000336^{*} B 79^{*} B 70^{*}\left(B 75^{\wedge} 2\right) / B 72 /\left(B 69^{\wedge} 5\right)$ & 0.283 & psi \\
\hline 81 & & & & \\
\hline 82 & Change in Gas at Common Outlet to Outside & & & Units \\
\hline 83 & Pressure in Cryostat & B4 & 19.75 & psig \\
\hline 84 & Pressure in Cryostat (conv.) & $=(B 83 / 14.696+1) * 1.01325$ & 2.375 & bars \\
\hline 85 & gAr Density @ 2.2 bars & -B9 & 11.77 & $\mathrm{mg} / \mathrm{cc}$ \\
\hline 86 & gAr Density @ 2.4 bars & $=B 10$ & 12.75 & $\mathrm{mg} / \mathrm{cc}$ \\
\hline 87 & gAr Density @ 2.375 \& Temp. & $=(B 84-2.2) / 0.2^{*}(B 86-B 85)+B 85$ & 12.627 & $\mathrm{mg} / \mathrm{cc}$ \\
\hline 88 & Temp. at Common Outlet & $=\left(\mathrm{B} 2^{*} \mathrm{~B} 17+84^{*} \mathrm{~B} 189\right) /(\mathrm{B} 17+\mathrm{B} 189)$ & 94 & $K$ \\
\hline 88 & > Pressure to Calculate Density & 1.5 & 1.5 & bars \\
\hline 90 & * gAr Density @ 1.5 bars \& New Temp. & 7.99 & 7.99 & $m g / c c$ \\
\hline 91 & I> gAr Viscosity @ 1.5 bars \& Now Temp. & 0.000078 & 0.000078 & $\mathrm{~g} / \mathrm{cm}-\mathrm{s}$ \\
\hline 92 & gAr Viscosity @ 1.5 bar (conv.) & $=891 * 100$ & 0.0078 & centipoise \\
\hline 93 & & & & \\
\hline
\end{tabular}




\section{Maximum ECS Rellef Flow Calculation 10/18/91}

\begin{tabular}{|c|c|c|c|c|}
\hline & A & B & C & D \\
\hline 94 & $\Delta P$ Across Relief Valve & & & Units \\
\hline 96 & $>$ Specific Heat Ratio (k) for Argon & -8168 & 1.75 & \\
\hline 97 & $>$ Area of $2^{n} \times 3^{n}$ Relief Valve & 2.29 & 2.29 & in^2 \\
\hline 98 & Flowing Temperature & $=1.8 * \mathrm{B2}$ & 173. & $\operatorname{deg} R$ \\
\hline 100 & $>$ Compressibility Factor & 1 & 1 & \\
\hline 101 & $>$ Nozzle Coefficient for type 93T & 0.939 & 0.939 & \\
\hline 104 & Critical Pressure (Pcr) & $-B 95 * B 102$ & 15.8 & psia \\
\hline 105 & Outlet Pressure (P2) (using delta p's) & $-14.696+\mathrm{B} 154+\mathrm{B} 133+\mathrm{B} 65$ & 25.43 & psia \\
\hline 106 & Pressure Ratio (P2"/P1) & $-\left(B 102-0.55^{*}\left((B 102-B 105)^{\wedge} 0.98\right)\right) / B 102$ & 0.885 & \\
\hline 107 & Theoretical Factor (F*) (using P2) & -SQAT $\left((B 96 /(B 96-1))^{*}\left(B 106^{\wedge}(2 / B 96)-B 106^{\wedge}((B 96+1) / B 96)\right)\right)$ & 0.321 & \\
\hline 108 & Max. Theoretical Relief Flow (using F*) & $-735^{*} \mathrm{~B} 97^{*} \mathrm{~B} 101^{*} \mathrm{~B} 102^{*} \mathrm{~B} 107^{*}$ SQRT(B103/B99/B100) & 7918 & $\mathrm{lb} \mathrm{m} / \mathrm{hr}$ \\
\hline 100 & Theoretical Percent of Relief Flow & $=\mathrm{B} 108 / \mathrm{B} 17$ & 0.4751 & \\
\hline 110 & Pressure Drop Across Relief Valve & $-B 102-B 105$ & 7.026 & psi \\
\hline
\end{tabular}




\begin{tabular}{|c|c|c|c|c|}
\hline & $\overline{\mathbf{A}}$ & $\mathbf{B}$ & $\mathbf{C}$ & $\mathbf{D}$ \\
\hline 112 & $\triangle \mathrm{P}$ Across Common Outlet to Cryocorner & & & Units \\
\hline 113 & > Inner Pipe Diameter & 0.355 & 0.355 & ft \\
\hline 114 & Inner Pipe Diameter (conv.) & $=B 113^{*} 12$ & 4.26 & in \\
\hline 115 & > Equivalent Length & 238 & 238 & $f t$ \\
\hline 116 & gAr Density @ 1.5 bar \& Now Temp. & $=\mathrm{BgO}$ & 7.99 & $\mathrm{mg} / \mathrm{cc}$ \\
\hline 117 & gAr Density @ 1.5 bar (conv.) & $-B 116 / 1000^{*} 62.428$ & 0.499 & $\mathrm{lbm} / \mathrm{ft}^{\wedge} 3$ \\
\hline 118 & > gN2 Gas Density @ 1.5 bar \& Now Temp. & 5.653 & 5.653 & $\mathrm{mg} / \mathrm{cc}$ \\
\hline 119 & gN2 Gas Density @ 1.5 bar (conv.) & $-B 118 / 1000^{\circ} 62.428$ & 0.353 & $\mathrm{Ibm} / \mathrm{ft}^{\wedge} \mathrm{3}$ \\
\hline 120 & Gas Mixture Density @1.5 bar & $=\left(B 17^{*} B 117+B 127^{*} B 119\right) / B 128$ & 0.474 & $1 \mathrm{bm} / \mathrm{ft}^{\wedge} 3$ \\
\hline 121 & JoAr Viscosity @ 1.5 bar \& Now Temp. & B91 & 0.000078 & $\mathrm{~g} / \mathrm{cm}-\mathrm{s}$ \\
\hline 122 & gAr Viscosity @ 1.5 bar (conv.) & $=8121 * 100$ & 0.0078 & centipoise \\
\hline 123 & $>$ gN2 Viscosity @ 1.5 bar \& New Temp. & 0.0000643 & 0.0000643 & $\mathrm{~g} / \mathrm{cm}-\mathrm{s}$ \\
\hline 124 & gN2 Viscosity @ 1.5 bar (conv.) & $=B 123 * 100$ & 0.00643 & centipoise \\
\hline 125 & Mixture Viscosity @1.5 bar & $=\left(\mathrm{B} 17^{*} \mathrm{~B} 122+\mathrm{B} 127^{*} \mathrm{~B} 124\right) / \mathrm{B} 128$ & 0.007563 & centipoise \\
\hline 126 & Max. Mass Flow of Argon Gas & $=B 17$ & 16666 & $\mathrm{Ibm} / \mathrm{hr}$ \\
\hline 127 & Max. Flow of Nitrogen Gas & $=\mathrm{B} 189$ & 3477 & $\mathrm{lbm} / \mathrm{hr}$ \\
\hline 128 & Mass Flow of Mixture & $=B 126+B 127$ & 20143 & $\mathrm{Ib} \mathrm{m} / \mathrm{hr}$ \\
\hline 129 & Reynolds Number & $=6.31^{*} \mathrm{~B} 128 /\left(\mathrm{B} 114^{*} \mathrm{~B} 125\right)$ & 3940000 & \\
\hline 130 & $>$ Relative Roughness $(e / D)$ & 0.0004 & 0.0004 & \\
\hline 131 & Friction Factor Guess & $=0.25^{*}\left(\operatorname{LOG}\left(B 130 / 3.7+5.74 /\left(B 129^{\wedge} 0.9\right)\right)\right)^{\wedge}-2$ & 0.0161 & \\
\hline 132 & Friction Factor & $=0.25^{*}\left(\operatorname{LOG}\left(B 130 / 3.7+2.51 /\left(\mathrm{B} 129^{*} B 131^{\wedge} 0.5\right)\right)\right)^{\wedge}-2$ & 0.0161 & \\
\hline 133 & Pressure Drop & $=0.00000336^{*} \mathrm{~B} 132^{*} \mathrm{~B} 115^{*}\left(\mathrm{~B} 128^{\wedge} 2\right) / \mathrm{B} 120 /\left(\mathrm{B} 114^{\wedge} 5\right)$ & 7.838 & psi \\
\hline 134 & & & & \\
\hline
\end{tabular}




\section{Maximum ECS Rellef Flow Calculation 10/18/91}

\begin{tabular}{|c|c|c|c|c|}
\hline & $\mathbf{A}$ & $\mathbf{B}$ & $\mathbf{C}$ & D \\
\hline 135 & $\Delta \mathrm{P}$ from Cryocorner to Outside & & & Units \\
\hline 136 & $>$ Inner Pipe Diameter & 0.53 & 0.53 & ft \\
\hline 137 & Inner Pipe Dlameter (conv.) & $=B 136^{*} 12$ & 6.36 & in \\
\hline 138 & $>$ Equivalent Length & 516 & 516 & ft \\
\hline 130 & gAr Gas Density @ 1.5 bar \& Naw Temp. & $-B 90$ & 7.99 & $\mathrm{mg} / \mathrm{cc}$ \\
\hline 140 & gAr Gas Density @ 1.5 bar (conv.) & $-8139 / 1000^{*} 62.428$ & 0.499 & $\mathrm{Ibm} / \mathrm{ft}^{\wedge} 3$ \\
\hline 141 & aN2 Gas Density @ 1.5 bar \& Now Temp. & $-B 118$ & 5.653 & $m g / c c$ \\
\hline 142 & gN2 Gas Density @ 1.5 bar (conv.) & $-B 141 / 1000^{*} 62,428$ & 0.353 & $\mathrm{lbm} / \mathrm{ft}^{\wedge} 3$ \\
\hline 143 & Gas Mixture Density @1.5 bar & $=\left(\mathrm{B} 17^{*} \mathrm{~B} 140+\mathrm{B} 189^{\circ} \mathrm{B} 142\right) / \mathrm{B} 149$ & 0.474 & $1 \mathrm{bm} / \mathrm{ft}^{\wedge} 3$ \\
\hline 144 & gAr Viscosity @ 1.5 bar \& New Temp. & $-B 121$ & 0.000078 & $\mathrm{~g} / \mathrm{cm}-\mathrm{s}$ \\
\hline 145 & gAr Viscosity @ 1.5 bar (conv.) & $=\mathrm{B} 144^{*} 100$ & 0.0078 & centipoise \\
\hline 146 & gN2 Viscosity @ 1.5 bar \& Now Temp. & $-\mathrm{B} 123$ & 0.0000643 & $\mathrm{~g} / \mathrm{cm}-\mathrm{s}$ \\
\hline 147 & gN2 Viscosity @ 1.5 bar (conv.) & $=\mathrm{B} 146 * 100$ & 0.00643 & centipoise \\
\hline 148 & Gas Mixture Viscosity @1.5 bar & $=\left(B 17^{*} B 145+B 189^{*} B 147\right) / B 149$ & 0.007563 & centipoise \\
\hline 149 & Max. Mass Flow of Gas Mixture & $-B 128$ & 20143 & $\mathrm{lb} \mathrm{m} / \mathrm{hr}$ \\
\hline 150 & Reynolds Number & $-6.31^{*} \mathrm{~B} 149 /\left(\mathrm{B} 137^{*} \mathrm{~B} 148\right)$ & 2640000 & \\
\hline 151 & $>$ Relative Roughness $(\theta / D)$ & 0.00027 & 0.00027 & \\
\hline 152 & Friction Factor Guess & $-0.25^{*}\left(\mathrm{LOG}\left(\mathrm{B} 151 / 3.7+5.74 /\left(\mathrm{B} 150^{\wedge} 0.9\right)\right)\right)^{\wedge}-2$ & 0.015 & \\
\hline 153 & Friction Factor & $=0.25^{*}\left(\operatorname{LOG}\left(B 151 / 3.7+2.51 /\left(B 150^{*} B 152^{\wedge} 0.5\right)\right)\right)^{\wedge}-2$ & 0.0149 & \\
\hline 154 & Pressure Drop & $=0.00000336^{*} \mathrm{~B} 153^{*} \mathrm{~B} 138^{*}\left(\mathrm{~B} 149^{\wedge} 2\right) / \mathrm{B} 143 /\left(\mathrm{B} 137^{\wedge} 5\right)$ & 2.13 & psi \\
\hline 155 & & & & \\
\hline 156 & Summation of Equivalent $\Delta \mathrm{PS}$ & & & Units \\
\hline 157 & Relief Valve Inlot Pressure Drop & $-\mathrm{B} 33$ & 1.993 & psi \\
\hline 158 & Rellef Valve Outlet Pressure Drop & $-\mathrm{B} 65$ & 0.764 & psi \\
\hline 159 & Relief Valve Pressure Drop & $-B 110$ & 7.026 & psi \\
\hline 160 & Relief Valve/Disk Branch & $=B 33+B 65+B 110$ & 9.782 & psi \\
\hline 161 & Rupture Disk Inlet Pressure Drop & $-\mathrm{B48}$ & 8.185 & psi \\
\hline 162 & Rupture Disk Outlet Pressure Drop & -880 & 0.283 & psi \\
\hline 163 & Rupture Disk Pressure Drop & $-\mathrm{B} 157+\mathrm{B} 158+\mathrm{B} 159-\mathrm{B} 161-\mathrm{B} 162$ & 1.313 & psi \\
\hline 164 & Common Outlet Pressure Drop & $=\mathrm{B} 133$ & 7.838 & psi \\
\hline 165 & Cryocorner to Outside Pressure Drop & $=\mathrm{B} 154$ & 2.13 & psi \\
\hline 166 & & & & \\
\hline
\end{tabular}




\section{Maximum ECS Rellef Flow Calculation 10/18/91}

\begin{tabular}{|c|c|c|c|c|}
\hline & A & B & C & D \\
\hline 167 & $\triangle P$ Across Rupture Disk & & & Units \\
\hline 168 & $>$ Argon Specific Heat Ratio $(\mathbf{k})$ & 1.745 & 1.745 & \\
\hline 169 & Critical Ratio & $=(2 /(B 168+1))^{\wedge}(B 168 /(B 168-1))$ & 0.476 & \\
\hline 170 & > Area of $3^{n}$ Rupture Disk & $=3.14159^{*}\left(3^{\wedge} 2\right) / 4$ & 7.069 & $\operatorname{lin}^{\wedge} 2$ \\
\hline 171 & Flow Through Rupture Disk & $=\mathrm{B} 43$ & 8766 & $\mathrm{lbm} / \mathrm{hr}$ \\
\hline 172 & Flowing Temperature & $-1.8^{*} \mathrm{~B} 2$ & 173 & $\operatorname{deg} R$ \\
\hline 173 & $\triangle$ ASME Cosfficient $(K)$ & 0.62 & 0.62 & \\
\hline 174 & Pressure Ratio (Pe/Po) & $=B 179 / B 176$ & 0.95 & \\
\hline 175 & gAr Flow Constant for Subsonic Flow(C1) & $=\operatorname{SQRT}\left(2^{*} 32.2 / 1545^{\circ}(\mathrm{B} 168 /(\mathrm{B} 168-1))^{*}\left(\mathrm{~B} 174^{\wedge}(2 / \mathrm{B} 168)-\mathrm{B} 17\right.\right.$ & 0.045 & \\
\hline 176 & Flowing Inlet Pressure (Po) & $-B 4+14.696-B 48$ & 26.26 & psia \\
\hline 177 & > Molocular Woight of Argon & 39.948 & 39.948 & $\mathrm{~g} / \mathrm{mol}$ \\
\hline 178 & Critical Pressure (Pcr) & $-B 169^{\circ} \mathrm{B} 176$ & 12.51 & psia \\
\hline 179 & Outlet Pressure (Pe) (using delta P's) & $=14.696+B 154+B 133+B 80$ & 24.95 & psia \\
\hline 180 & Pressure Drop Across Rupture Disk & -B176-B179 & 1.313 & psi \\
\hline 181 & Maximum Theoretical Rupture Disk Flow & $=\mathrm{B} 170^{\circ} \mathrm{B} 173^{*} \mathrm{~B} 175^{*} \mathrm{~B} 176^{*} \mathrm{SQRT}(\mathrm{B} 177 / \mathrm{B} 172)^{*} 60^{*} 60$ & 8897 & $1 \mathrm{bm} / \mathrm{hr}$ \\
\hline 182 & Actual Rupture Disk Flow & $-\mathrm{B} 43$ & 8766 & $\mathrm{Ibm} / \mathrm{hr}$ \\
\hline \multicolumn{5}{|c|}{183} \\
\hline 184 & Maximum Flow from Condensers & & & units \\
\hline 185 & > Max. Flow of Liquid Nitrogen & 9.3 & 9.3 & gpm \\
\hline 186 & Max. Flow of Liquid Nitrogen (conv.) & $-B 185 / 7.48$ & 1.24 & ft^3/min \\
\hline 187 & Density of LN2 @ $3.5 \mathrm{~atm}$ & 0.747 & 0.747 & g/ce \\
\hline 188 & Density of LN2 (conv.) & $=B 187^{*} 62.4$ & 46.6128 & $16 \mathrm{~m} / \mathrm{ft}^{\wedge} 3$ \\
\hline 180 & Mass Flow of LN2 & $-B 186^{*} B 188^{*} 60$ & 3477 & $16 \mathrm{~m} / \mathrm{hr}$ \\
\hline \multicolumn{5}{|c|}{190} \\
\hline \multirow{2}{*}{\multicolumn{5}{|c|}{$\begin{array}{l}191 \text { Notea: } \\
192 \text { t } \\
\text { indicates that this value must be changed for a }\end{array}$}} \\
\hline \multirow{2}{*}{\multicolumn{3}{|c|}{$1922>$ indicates that this value must be changed for a }} & & \\
\hline \multirow{2}{*}{\multicolumn{5}{|c|}{\begin{tabular}{|l|l|}
193 & now flowrate \\
194 & \\
\end{tabular}}} \\
\hline & & & & \\
\hline \multicolumn{5}{|c|}{$105>$ Indicates varlable not requiring change for naw } \\
\hline \multicolumn{5}{|c|}{196 flowrates } \\
\hline \multicolumn{5}{|c|}{187} \\
\hline \multicolumn{5}{|c|}{198 (conv.) indicates the previous value converted to new } \\
\hline 189 & units & & & \\
\hline
\end{tabular}


As with the ECN, the total mass flow for the ECS can be calculated from line 108 for the maximum relief valve flow, and line 181 for the maximum rupture disk flow:

$\rho\left(q_{\mathrm{rel}}\right)_{\max }=7918 \frac{1 b_{m}}{h_{r}}($ relief valve $)+8897 \frac{1 b_{m}}{h r}($ rupture disk $)=16,815 \frac{1 b_{m}}{h_{r}}$

Now the ECN and ECS relief piping flowrates can be compared:

$\frac{q_{\mathrm{rel}}}{\mathrm{q}_{\mathrm{rel}}}=\frac{\rho q_{\mathrm{rel}}^{E C S}}{\rho q_{\mathrm{rel}}^{E C N}}=\frac{16,815 \frac{1 b_{\mathrm{m}}}{\mathrm{h} \mathrm{r}}}{15,198 \frac{1 b_{\mathrm{m}}}{\mathrm{h} \mathrm{r}}}=1.106$

The maximum relief outlet flow from the ECS is greater than the maximum relief outlet flow from the ECN by about $10 \%$.

\section{Conclusion}

Since the flowrate out of the ECS relative to the flowrate out of the ECN (1.106 times ECN) is greater than the flowrate into the ECS relative to the flowrate into the ECN (1.054 times ECN), the ECN is the flowrate limiting vessel. 


\section{Relief Valve Capacity for Filling ECN at Maximum Operating Temperature}

Although the maximum temperature calculated by this engineering note is $290 \mathrm{~K}$, the spreadsheet requires flow through both the relief valve and the rupture disk. Although this situation satifies safety conditions regarding the overpressurization of the vessel, in reality, the operating procedures should limit the temperature to a much lower value, to prevent the rupture disk from bursting. This section calculates the temperature at which the maximum flow from the argon dewar requires only the relief valve, and not the rupture disk.

The procedure is the same as before, so the same spreadsheet is used. The only difference is that in the section, "Conversion of Liquid to Gas at Module Temp.", line 18 is not guessed through iteration, but is set initially to $100 \%$. This forces all of the flow through the relief valve, and all of the sections related to the rupture disk, including the pressure drops before and after the rupture disk, are essentially excluded from the calculation. Rather than physically remove these sections, they remain in the spreadsheet, but have no effect on the temperature. The bulk temperature is guessed as before, and iterations proceed, changing the various gas properties of argon and nitrogen each time, until a suitable temperature is found. The conclusion was that with a maximum flow of $12.3 \mathrm{gpm}$, as calculated previously, the maximum module temperature at which only the relief valve is required is at least $110 \mathrm{~K}$. Note that the theoretical percent of relief flow (line 109) is greater than $100 \%$, indicating that the relief capacity is above the inlet flow. This can be verified by comparing line 98 (actual flow) and line 108 (maximum theoretical flow). 
Maximum Module Temperature - Lar Dewar to ECN - Rellef Valve Only 10/18/91

\begin{tabular}{|c|c|c|c|c|}
\hline & A & B & c & D \\
\hline 1 & Conversion of Liquid to Gas at Module Temp. & & & Units \\
\hline 2 & "> Bulk Temp. of Modules & 110 & 110 & $\mathbf{K}$ \\
\hline 3 & $>$ Max. Flow of Liquid Argon to Cryostat & 12.3 & 12.3 & gpm \\
\hline 4 & $>$ Pressure in Cryostat & 19.75 & 19.75 & psig \\
\hline 5 & Pressure in Cryostat (conv.) & $=(B 4 / 14.696+1) * 1.01325$ & 2.375 & bars \\
\hline 6 & > IAr Density @ 2.2 bars & 1.342421 & 1.34 & g/cc \\
\hline 7 & $>$ IAr Density @ 2.4 bars & 1.335861 & 1.34 & g/cc \\
\hline 8 & |Ar Density @ 2.375 bars & $=(B 5-2.2) / 0.2^{*}(B 7-B 6)+B 6$ & 1.337 & g/cc \\
\hline$\theta$ & - > gAr Density @ 2.2 bars & 9.979 & 9.979 & $\mathrm{mg} / \mathrm{cc}$ \\
\hline 10 & -> gAr Density @ 2.4 bars & 10.922 & 10.922 & $\mathrm{mg} / \mathrm{cc}$ \\
\hline 11 & gAr Density @ 2.375 \& Temp. & $=(B 5-2.2) / 0.2^{*}(B 10-B 9)+B 9$ & 10.804 & $\mathrm{mg} / \mathrm{cc}$ \\
\hline 12 & gAr Density @ 2.375 bars (conv.) & $=\mathrm{B} 11 / 1000^{*} 62.428$ & 0.674 & $16 \mathrm{~m} / \mathrm{ft}^{\wedge} \mathbf{3}$ \\
\hline 13 & Maximum Flow of Argon Gas & $=\mathrm{B} 3 * \mathrm{~B} 8 / \mathrm{B} 11 * 1000$ & 1522 & gpm \\
\hline 14 & Maximum Flow of Argon Gas (conv.) & $=B 13^{*} 0.13368$ & 203 & $\mathrm{cfm}$ \\
\hline 15 & Air Equivalent Flow @STP & $=6.32^{*} \mathrm{~B} 17^{*} 356 / \mathrm{B}^{\prime} 6^{*} \mathrm{SQRT}\left(\mathrm{B} 99 /\left(520^{*} \mathrm{~B} 177^{*} 28.97\right)\right)$ & 881 & scfm air \\
\hline 16 & Specific Heat Constant, C, for Ar & $=520^{*} \mathrm{SQRT}\left(\mathrm{B} 168^{*}(2 /(\mathrm{B} 168+1))^{\wedge}((\mathrm{B} 168+1) /(\mathrm{B} 168-1))\right)$ & 381 & \\
\hline 17 & Max. Mass Flow of Argon Gas & $=B 14^{*} B 12 * 60$ & 8232 & $16 \mathrm{~m} / \mathrm{hr}$ \\
\hline 18 & > Percent of Mass Flow to Relief Valve & 1 & 1 & \\
\hline 10 & & & & \\
\hline 20 & $\Delta \mathrm{P}$ Across Relief Valve Inlet & & & Units \\
\hline 21 & $>$ Inner Pipe Diameter & 0.206 & 0.206 & $f t$ \\
\hline 22 & Inner Pipe Diameter (conv.) & $=\mathrm{B} 21 * 12$ & 2.472 & in \\
\hline 23 & $>$ Equivalent Length & 38 & 38 & ft \\
\hline 24 & Ar Gas Density @ 2.375 \& Tomp. & $=(B 5-2.2) / 0.2^{*}(\mathrm{~B} 10-\mathrm{B} 9)+\mathrm{B} 9$ & 10.804 & $\mathrm{mg} / \mathrm{cc}$ \\
\hline 25 & Ar Gas Density @ 2.375 bars (conv.) & $=B 24 / 1000^{*} 62.428$ & 0.674 & $1 \mathrm{bm} / \mathrm{ft}^{\wedge} \mathbf{3}$ \\
\hline 26 & > gAr Viscosity @ 2.4 bars \& Temp. & 0.0000909 & 0.0000909 & $\mathrm{~g} / \mathrm{cm}-\mathrm{s}$ \\
\hline 27 & gAr Viscosity @ 2.4 bars (conv.) & $=B 26 * 100$ & 0.00909 & centipoise \\
\hline 28 & Max. Mass Flow to Relief Valve & $=B 17^{*} B 18$ & 8232 & $\mathrm{lbm} / \mathrm{hr}$ \\
\hline 29 & Reynolds Number & $=6.311^{\circ} \mathrm{B} 28 /\left(\mathrm{B} 22^{*} \mathrm{~B} 27\right)$ & 2310000 & \\
\hline 30 & > Relative Roughness ( $\theta / \mathrm{D})$ & 0.0007 & 0.0007 & \\
\hline 31 & Friction Factor Guess & $=0.25^{*}\left(\operatorname{LOG}\left(\mathrm{B} 30 / 3.7+5.74 /\left(\mathrm{B} 29^{\wedge} 0.9\right)\right)\right)^{\wedge}-2$ & 0.0183 & \\
\hline 32 & Friction Factor & $=0.25^{\circ}\left(\operatorname{LOG}\left(B 30 / 3.7+2.51 /\left(B 29^{*} B 31^{\wedge} 0.5\right)\right)\right)^{\wedge}-2$ & 0.0182 & \\
\hline 33 & Pressure Drop & $=0.00000336^{*} \mathrm{~B} 32^{*} \mathrm{~B} 23^{*}\left(\mathrm{~B} 28^{\wedge} 2\right) / \mathrm{B} 25 /\left(\mathrm{B} 22^{\wedge} 5\right)$ & 2.531 & psi \\
\hline 34 & & & & \\
\hline
\end{tabular}




\begin{tabular}{|c|c|c|c|c|}
\hline & $\bar{A}$ & $\mathbf{B}$ & $\mathbf{C}$ & D \\
\hline 35 & $\Delta \mathrm{P}$ Across Rupture Disk Inlet & & & Units \\
\hline 36 & Inner Pipe Diameter (conv.) & $-837 / 12$ & 0.172 & $f t$ \\
\hline 37 & $>$ Inner Pipe Diameter & 2.067 & 2.067 & in \\
\hline 38 & $>$ Equivalent Length & 49 & 49 & $1 t$ \\
\hline 38 & gAr Density @ 2.375 \& Tomp. & $=(B 5-2.2) / 0.2^{\circ}(B 10-89)+B 9$ & 10.804 & $\mathrm{mg} / \mathrm{cc}$ \\
\hline 40 & gAr Density @ 2.375 bars (conv.) & $=B 39 / 1000^{*} 62.428$ & 0.674 & $1 \mathrm{bm} / \mathrm{ft}^{\wedge} 3$ \\
\hline 41 & gAt Viscosity @ 2.4 bars & $-B 26$ & 0.0000909 & $\mathrm{~g} / \mathrm{cm}-\mathrm{s}$ \\
\hline 42 & gAr Vlscosity @ 2.4 bars (conv.) & $=B 41^{*} 100$ & 0.00909 & centipoise \\
\hline 43 & Max. Mass Flow to Rupture Disk & $=B 17^{\circ}(1-B 18)$ & 0 & $\mathrm{lbm} / \mathrm{hr}$ \\
\hline 44 & Reynolds Number & $=6.31^{\circ} \mathrm{B} 43 /\left(\mathrm{B} 37^{\circ} \mathrm{B} 42\right)$ & 0 & \\
\hline 45 & $>$ Relative Roughness $(\theta / D)$ & 0.0009 & 0.0009 & \\
\hline 46 & Friction Factor Guess & 0 & 0 & \\
\hline 47 & Friction Factor & 0 & 0 & \\
\hline 48 & Pressure Drop & 0 & to & psi \\
\hline 51 & & & & \\
\hline 52 & $\triangle P$ Across Relief Valve Outlet & & & Units \\
\hline 53 & Inner Pipe Diameter (conv.) & $=B 54 / 12$ & 0.272 & $\mathrm{ft}$ \\
\hline 54 & $>$ Inner Plpe Diameter & 3.26 & 3.26 & in \\
\hline 55 & $>$ Equivalont Length & 51 & 51 & ft \\
\hline 56 & $">$ gAr Density @ 2.0 bar \& Temp. & 9.04 & 9.04 & $\mathrm{mg} / \mathrm{cc}$ \\
\hline 57 & gAr Density @ 2.0 bar (conv.) & m556/1000*62.428 & 0.564 & $16 \mathrm{~m} / \mathrm{ft}^{\wedge} 3$ \\
\hline 58 & $>$ gAr Viscosity @ 2.0 bar \& Temp. & 0.0000907 & 0.0000907 & $\mathrm{~g} / \mathrm{cm}-\mathrm{s}$ \\
\hline 50 & gAr Viscosity @ 2.0 bar (conv.) & $=858 * 100$ & 0.00907 & centipoise \\
\hline 60 & Max. Mass Flow to Relief Valve & -828 & 8232 & $16 \mathrm{~m} / \mathrm{hr}$ \\
\hline 61 & Reynolds Number & $=6.31^{\circ} \mathrm{B} 60 /\left(\mathrm{B} 54^{\circ} \mathrm{B} 59\right)$ & 1760000 & \\
\hline 62 & $>$ Relative Roughness $(\mathrm{e} / \mathrm{D})$ & 0.00055 & 0.00055 & \\
\hline 63 & Friction Factor Guess & $=0.25^{\circ}\left(\operatorname{LOG}\left(B 62 / 3.7+5.74 /\left(B 61^{\wedge} 0.9\right)\right)\right)^{\wedge}-2$ & 0.0174 & \\
\hline 64 & Friction Factor & $=0.25^{\circ}\left(\mathrm{LOG}\left(\mathrm{B} 62 / 3.7+2.51 /\left(\mathrm{B} 61^{\circ} \mathrm{B} 63^{\wedge} 0.5\right)\right)\right)^{\wedge}-2$ & 0.0173 & \\
\hline 65 & Pressure Drop & $=0.000000336^{*} \mathrm{~B} 64^{*} \mathrm{~B} 55^{*}\left(\mathrm{~B} 60^{\wedge} 2\right) / \mathrm{B} 57 /\left(\mathrm{B} 54^{\wedge} 5\right)$ & 0.969 & psi \\
\hline 68 & & & & \\
\hline
\end{tabular}




\begin{tabular}{|c|c|c|c|c|}
\hline & $\mathbf{A}$ & $\mathbf{B}$ & C & $\mathbf{D}$ \\
\hline 67 & $\triangle P$ Across Rupture Disk Outlet & & & Units \\
\hline 68 & Inner Pipe Diameter (conv.) & $=869 / 12$ & 0.18 & $f t$ \\
\hline 69 & $>$ Inner Pipe Diameter & 2.157 & 2.157 & $\ln$ \\
\hline 70 & $>$ Equivalent Length & 1.75 & 1.75 & ft \\
\hline 71 & gr Density \& 2.0 bar \& Temp. & $=\mathrm{B56}$ & 9.04 & $\mathrm{mg} / \mathrm{cc}$ \\
\hline 72 & gAr Density @ 2.0 bar (conv.) & $=B 71 / 1000^{*} 62.428$ & 0.564 & $1 \mathrm{bm} / \mathrm{ft}^{\wedge} \mathrm{3}$ \\
\hline 73 & gAr Viscosity @ 2.0 bar \& Temp. & $=\mathrm{B} 58$ & 0.0000907 & $\mathrm{~g} / \mathrm{cm}-\mathrm{s}$ \\
\hline 74 & oAr Viscosity @2.0 bar (conv.) & $=873 * 100$ & 0.00907 & Centipoise \\
\hline 75 & Max. Mass Flow to Rupture Disk & $\mathrm{BB} 43$ & 0 & $1 \mathrm{bm} / \mathrm{hr}$ \\
\hline 76 & Reynolds Number & $=6.31^{*} \mathrm{B75} /(\mathrm{B69} \cdot \mathrm{B} 74)$ & 0 & \\
\hline 77 & - Relative Roughness (e/D) & 0.0009 & 0.0009 & \\
\hline 78 & Friction Factor Guess & 0 & 0 & \\
\hline 79 & Friction Factor & 0 & 0 & \\
\hline 80 & Pressure Drop & 0 & 0 & psi \\
\hline 81 & & & & \\
\hline 82 & Change in Gas at Common Outlet to Outside & & & Units \\
\hline 83 & Pressure in Cryostat & -B4 & 19.75 & psig \\
\hline 84 & Pressure in Cryostat (conv.) & $=(B 83 / 14.696+1) * 1.01325$ & 2.375 & bars \\
\hline 85 & gAr Density @ 2.2 bars & $=\mathrm{B9}$ & 9.979 & $\operatorname{mg} / \mathrm{cc}$ \\
\hline 86 & gAr Density Q 2.4 bars & $=810$ & 10.922 & $m g / c c$ \\
\hline 87 & gAr Density @ 2.375 \& Temp. & $=(\mathrm{B} 84-2.2) / 0.2^{*}(\mathrm{~B} 86-\mathrm{B} 85)+\mathrm{B85}$ & 10.804 & $\mathrm{mg} / \mathrm{cc}$ \\
\hline 88 & Temp. at Common Cutlet & $=\left(\mathrm{B} 2^{*} \mathrm{~B} 17+84^{\circ} \mathrm{B} 189\right) /(\mathrm{B} 17+\mathrm{B} 189)$ & 100 & $K$ \\
\hline 80 & > Pressure to Calculate Density & 1.5 & 1.5 & bars \\
\hline 90 & > gAr Density $\propto 1.5$ bars \& Now Temp. & 7.458 & 7.458 & $\mathrm{mg} / \mathrm{cc}$ \\
\hline 91 & > gAr Viscosity \& 1.5 bars \& New Temp. & 0.0000826 & 0.0000826 & $\mathrm{~g} / \mathrm{cm}-\mathrm{s}$ \\
\hline 02 & gAr Viscosity @ 1.5 bar (conv.) & $=891 * 100$ & 0.00826 & Centipoise \\
\hline 93 & & & & \\
\hline
\end{tabular}




\begin{tabular}{|c|c|c|c|c|}
\hline & A & B & C & D \\
\hline 94 & $\Delta P$ Across Relief Valve & & & Units \\
\hline 95 & $>$ Critical Ratio (Pcr/P1) for Argon & 0.487 & 0.487 & \\
\hline 96 & > Specific Heat Ratio (k) for Argon & $-B 168$ & 1.72 & \\
\hline 97 & $>$ Area of $2^{*} \times 3^{n}$ Rellef Valve & 2.29 & 2.29 & in^2 \\
\hline 98 & Flow Through Relief Valve & $-B 28$ & 8232 & $\mathrm{bm} / \mathrm{hr}$ \\
\hline 99 & Flowing Temperature & $=1.8^{*} \mathrm{B2}$ & 198 & $\operatorname{deg} R$ \\
\hline 100 & $>$ Compressibility Factor & 1 & 1 & \\
\hline 101 & $>$ Nozzle Coefficient for type 93T & 0.939 & 0.939 & \\
\hline 102 & Flowing Inlat Pressure (P1) & $=\mathrm{B} 4+14.696-\mathrm{B} 33$ & 31.91 & psia \\
\hline 103 & $>$ Molocular Woight of Argon & 39.948 & 39.95 & $\mathrm{~g} / \mathrm{mol}$ \\
\hline 104 & Critical Pressure (Por) & $-B 95 \times B 102$ & 15.54 & psia \\
\hline 105 & Outlet Pressure (P2) (using delta $\mathrm{p}^{\prime}$ 's) & $=14.696+B 154+B 133+B 65$ & 21.22 & psia \\
\hline 106 & Prossure Ratio (P2*/P1) & $=\left(B 102-0.55^{*}\left((\mathrm{~B} 102-\mathrm{B} 105)^{\wedge} 0.98\right)\right) / \mathrm{B} 102$ & 0.824 & \\
\hline 107 & Theoretical Factor (F*) (using $\mathrm{P}_{2}$ ) & -SQRT $\left((B 96 /(B 96-1))^{*}\left(B 106^{\wedge}(2 / B 96)-B 106^{\wedge}((B 96+1) / B 96)\right)\right)$ & 0.385 & \\
\hline 108 & Max. Theoretical Relief Flow (using $F^{*}$ ) & $=735^{*} \mathrm{~B} 97^{*} \mathrm{~B} 101^{*} \mathrm{~B} 102^{*} \mathrm{~B} 107^{*} \mathrm{SQRT}(\mathrm{B} 103 / \mathrm{B} 99 / \mathrm{B} 100)$ & 8723 & $\mathrm{lb} \mathrm{m} / \mathrm{hr}$ \\
\hline 100 & Theoretical Percent of Relief Flow & $-\mathrm{B} 108 / \mathrm{B} 17$ & 1.0596 & \\
\hline 110 & Pressure Drop Across Rellef Valve & $=B 102-B 105$ & 10.69 & psi \\
\hline 1111 & & & & \\
\hline
\end{tabular}




\begin{tabular}{|c|c|c|c|c|}
\hline & $\mathbf{A}$ & $\mathbf{B}$ & C & $\mathbf{D}$ \\
\hline 112 & $\triangle P$ Across Common Outlet to Platform Bayonet & & & Units \\
\hline 113 & $>$ Inner Pipe Diameter & 0.355 & 0.355 & $f t$ \\
\hline 114 & Inner Pipe Diameter (comv.) & $=8113^{* 12}$ & 4.26 & in \\
\hline 115 & $>$ Equivalent Length & 273 & 273 & $f t$ \\
\hline 116 & gAr Density $Q 1.5$ bar \& New Temp. & $\mathrm{B} 90$ & 7.458 & $m g / c c$ \\
\hline 117 & gAr Density @ 1.5 bar (conv.) & $-8116 / 1000^{*} 62.428$ & 0.466 & $1 \mathrm{bm} / \mathrm{ft}^{\wedge} 3$ \\
\hline 118 & 2 gN2 Gas Density 91.5 bar \& New Temp. & 4.76381 & 4.764 & $\mathrm{mg} / \mathrm{cc}$ \\
\hline 119 & gN2 Gas Density @ 1.5 bar (conv.) & $=B 118 / 1000^{\circ} 62.428$ & 0.297 & $1 \mathrm{bm} / \mathrm{ft}^{\wedge} \mathrm{3}$ \\
\hline 120 & Gas Mixture Density @1.5 bai & $=\left(\mathrm{B} 17^{*} \mathrm{~B} 117+\mathrm{B} 127^{*} \mathrm{~B} 119\right) / \mathrm{B} 128$ & 0.403 & $1 \mathrm{bm} / \mathrm{ft}^{\wedge} \mathrm{3}$ \\
\hline 121 & gAr Viscosity @ 1.5 bar \& Now Tomp. & $=891$ & 0.0000826 & $0 / \mathrm{cm}-\mathrm{s}$ \\
\hline 122 & gir Viscosity @ 1.5 bar (conv.) & $=8121^{* 100}$ & 0.00826 & centipoise \\
\hline 123 & $>$ gN2 Viscosity @ 1.5 bar \& New Temp. & 0.00007488 & 0.00007488 & $\mathrm{~g} / \mathrm{cm}-\mathrm{s}$ \\
\hline 124 & gN2 Viscosity $@ 1.5$ bar (conv.) & $-B 123^{*} 100$ & 0.007488 & centipoise \\
\hline 125 & Mixture Viscosity @1.5 bar & $=\left(\mathrm{B} 17^{*} \mathrm{~B} 122+\mathrm{B} 127^{*} \mathrm{~B} 124\right) / \mathrm{B} 128$ & 0.007973 & centipoise \\
\hline 120 & Max. Mass Flow of Argon Gas & $-\mathrm{B} 17$ & 8232 & $\mathrm{lbm} / \mathrm{hr}$ \\
\hline 127 & Max. Flow of Nitrogen Gas & $-B 189$ & 4861 & Ibm/hr \\
\hline 128 & Mass Flow of Mixture & $=\mathrm{B} 126+\mathrm{B} 127$ & 13093 & $16 \mathrm{~m} / \mathrm{hr}$ \\
\hline 120 & Reynolds Number & $=6.31^{\circ} \mathrm{B} 128 /\left(\mathrm{B} 114^{\circ} \mathrm{B} 125\right)$ & 2430000 & \\
\hline 130 & 2 Relative Rouphness (e/D) & 0.0004 & 0.0004 & \\
\hline 131 & Friction Factor Guess & $=0.25^{*}\left(\operatorname{LOG}\left(\mathrm{B} 130 / 3.7+5.74 /\left(\mathrm{B} 129^{\wedge} 0.9\right)\right)\right)^{\wedge}-2$ & 0.0162 & \\
\hline 132 & Friction Factor & $=0.25^{\star}\left(\mathrm{LOG}\left(\mathrm{B} 130 / 3.7+2.51 /\left(\mathrm{B} 129^{\star} \mathrm{B} 131^{\wedge} 0.5\right)\right)\right)^{\wedge}-2$ & 0.0161 & \\
\hline 133 & Pressure Drop & $=0.00000336^{*} \mathrm{~B} 132^{*} \mathrm{~B} 115^{\circ}\left(\mathrm{B} 128^{\wedge} 2\right) / \mathrm{B} 120 /\left(\mathrm{B} 11^{\wedge} 5\right)$ & 4.489 & |psi \\
\hline 134 & & & & \\
\hline
\end{tabular}




\begin{tabular}{|c|c|c|c|c|}
\hline & $A$ & $\mathbf{B}$ & $\mathbf{c}$ & $\mathbf{D}$ \\
\hline 135 & $\Delta \mathrm{P}$ from Platform Bayonet to Outside & & & Units \\
\hline 136 & $>$ Inner Pipe Diameter & 0.53 & 0.53 & ft \\
\hline 137 & Inner Pipe Diameter (conv.) & $-B 136^{* 12}$ & 6.36 & in \\
\hline 138 & $\geq$ Equivalent Length & 516 & 516 & $f t$ \\
\hline 130 & gar Gas Density @ 1.5 bar \& Now Temp. & $-B 90$ & 7.458 & $\mathrm{mg} / \mathrm{cc}$ \\
\hline 140 & gAr Gas Density @ 1.5 bar (conv.) & $=B 139 / 1000^{*} 62.428$ & 0.466 & $1 \mathrm{bm} / \mathrm{ft} \mathrm{t}^{\wedge} 3$ \\
\hline 141 & gN2 Gas Density \& 1.5 bar \& New Temp. & $=B 118$ & 4.764 & $\mathrm{mg} / \mathrm{cc}$ \\
\hline 142 & gN2 Gas Density @ 1.5 bar (conv.) & $=\mathrm{B} 141 / 1000^{*} 62.428$ & 0.297 & $1 \mathrm{bm} / \mathrm{ft}^{\wedge} 3$ \\
\hline 143 & Gas Mixture Densty $@ 1.5$ bar & $=\left(\mathrm{B} 17^{\circ} \mathrm{B} 140+\mathrm{B} 189^{\circ} \mathrm{B} 142\right) / \mathrm{B} 149$ & 0.403 & $\mathrm{lbm} / \mathrm{ft}^{\wedge} 3$ \\
\hline 144 & ar Viscosity \& 1.5 bar \& Now Tomp. & B121 & 0.0000826 & g/cm-s \\
\hline 145 & gAr Viscosity \& 1.5 bar (conv.) & $=8144 * 100$ & 0.00826 & centipoise \\
\hline 146 & gN2 Viscosity @ 1.5 bar \& New Temp. & $=\mathrm{B123}$ & 0.00007488 & $\mathrm{~g} / \mathrm{cm}-\mathrm{s}$ \\
\hline 147 & gN2 Viscosity @ 1.5 bar (conv.) & $=8146 * 100$ & 0.007488 & centipoise \\
\hline 148 & Gas Mixture Viscosity @1.5 bar & $=\left(\mathrm{B} 17^{\circ} \mathrm{B} 145+\mathrm{B} 189^{\circ} \mathrm{B} 147\right) / \mathrm{B} 149$ & 0.007973 & centipoise \\
\hline 140 & Max. Mass Flow of Gas Mixture & $=\mathrm{B} 128$ & 13093 & $\mathrm{lbm} / \mathrm{hr}$ \\
\hline 150 & Reynolds Number & $=6.311^{\circ} \mathrm{B} 149 /\left(\mathrm{B} 137^{\circ} \mathrm{B} 148\right)$ & 1630000 & \\
\hline 151 & > Rolative Roughnoss (e/D) & 0.00027 & 0.00027 & \\
\hline 152 & Friction Factor Guess & $=0.25^{*}\left(\operatorname{LOG}\left(B 151 / 3.7+5.74 /\left(\mathrm{B} 150^{\wedge} 0.9\right)\right)\right)^{\wedge}-2$ & 0.0152 & \\
\hline 153 & Friction Factor & $=0.25^{\prime \prime}\left(\operatorname{LOG}\left(B 151 / 3.7+2.51 /\left(B 150^{\circ} B 152^{\wedge} 0.5\right)\right)\right)^{\wedge}-2$ & 0.0151 & \\
\hline 154 & Pressure Drop & $=0.00000336^{\circ} \mathrm{B} 153^{*} \mathrm{~B} 138^{*}\left(\mathrm{~B} 149^{\wedge} 2\right) / \mathrm{B} 143 /\left(\mathrm{B} 137^{\wedge} 5\right)$ & 1.07 & psi \\
\hline 153 & & & & \\
\hline 156 & Summation of Equivalent $\Delta \mathrm{PS}$ & & & Units \\
\hline 157 & Relief Valve Inlet Pressure Drop & $=833$ & 2.531 & $|p s|$ \\
\hline 158 & Rellef Valve Outlet Pressure Drop & $=865$ & 0.969 & psi \\
\hline 159 & Relief Valve Pressure Drop & $=19.75-\mathrm{B} 157-(\mathrm{B} 165+\mathrm{B} 164+\mathrm{B} 158)$ & 10.69 & $|p s|$ \\
\hline 180 & Reliof Valve/Oisk Branch & $-\mathrm{B} 33+\mathrm{B} 65+\mathrm{B} 110$ & 14.19 & psi \\
\hline 161 & Rupture Disk Inlet Pressure Drop & $=848$ & 0 & psi \\
\hline 162 & Rupture Disk Outlet Pressure Drop & $=B 80$ & 0 & psi \\
\hline 163 & Rupture Disk Pressure Drop & 0 & 0 & $|p s|$ \\
\hline 164 & Common Outlet Pressure Drop & -8133 & 4.489 & psi \\
\hline 165 & Platform to Outside Pressure Drop & $=8154$ & 1.07 & psi \\
\hline 166 & & & & \\
\hline
\end{tabular}




\begin{tabular}{|c|c|c|c|c|}
\hline & $\mathbf{A}$ & $\mathbf{B}$ & C & D \\
\hline 167 & $\triangle P$ Across Rupture Disk & & & Units \\
\hline 168 & $>$ Argon Specific Heat Ratio (k) & 1.7186 & 1.7186 & \\
\hline 168 & Critical Ratio & $=(2 /(\mathrm{B} 168+1))^{\wedge}(\mathrm{B} 168 /(\mathrm{B} 168-1))$ & 0.48 & \\
\hline 170 & - Area of 3" Rupture Disk & $-3.14159^{*}\left(3^{\wedge} 2\right) / 4$ & 7.069 & $\operatorname{in}^{\wedge} 2$ \\
\hline 171 & Flow Through Rupture Disk & $-\mathrm{B} 43$ & 0 & $1 \mathrm{bm} / \mathrm{hr}$ \\
\hline 172 & Flowing Temperature & $=1.8^{*} \mathrm{B2}$ & 198 & $\operatorname{deg} R$ \\
\hline 173 & $>$ ASME Coefficient $(\mathrm{K})$ & 0.62 & 0.62 & \\
\hline 174 & Pressure Ratio (Pe/Po) & $=\mathrm{B} 179 / \mathrm{B} 176$ & 0.588 & \\
\hline 175 & gAr Flow Constant for Subsonic Flow(C1) & $=\operatorname{SQRT}\left(2^{*} 32.2 / 1545^{*}(\mathrm{~B} 168 /(\mathrm{B} 168-1))^{*}\left(\mathrm{~B} 174^{\wedge}(2 / \mathrm{B} 168)-\mathrm{B} 174\right.\right.$ & 0.103 & \\
\hline 176 & Flowing Inlet Pressure (Po) & $-84+14.696-B 48$ & 34.45 & psia \\
\hline 177 & > Molecular Weight of Argon & 39.948 & 39.948 & $\mathrm{~g} / \mathrm{mol}$ \\
\hline 178 & Critical Pressure (Pcr) & $=\mathrm{B} 169^{*} \mathrm{~B} 176$ & 16.53 & psia \\
\hline 179 & Outlot Pressure (Pe) (using delta p's) & $=14.696+\mathrm{B} 154+\mathrm{B} 133+\mathrm{B} 80$ & 20.26 & psia \\
\hline 180 & Pressure Drop Across Rupture Disk & 0 & 0 & psi \\
\hline 181 & Maximum Theoretical Rupture Disk Flow & $-\mathrm{B} 170^{*} \mathrm{~B} 173^{*} \mathrm{~B} 175^{*} \mathrm{~B} 176^{*} \mathrm{SORT}(\mathrm{B} 177 / \mathrm{B} 172)^{*} 60^{*} 60$ & 25250 & $1 \mathrm{bm} / \mathrm{hr}$ \\
\hline 182 & Actual Rupture Disk Flow & $-\mathrm{B} 43$ & 0 & Ibm/hr \\
\hline \multicolumn{5}{|c|}{183} \\
\hline 184 & Maximum Flow from Condensers & & & units \\
\hline 185 & $>$ Max. Flow of Liquid Nitrogen & 13 & 13 & gpm \\
\hline 186 & Max. Flow of Liquid Nitrogen (conv.) & $-8185 / 7.48$ & 1.74 & $\mathrm{ft}^{\wedge} \mathrm{3} / \mathrm{min}$ \\
\hline 187 & Density of LN2 @ $3.5 \mathrm{~atm}$ & 0.747 & 0.747 & $g / c c$ \\
\hline 188 & Density of LN2 (conv.) & $-B 187^{*} 62.4$ & 46.6128 & $1 \mathrm{bm} / \mathrm{ft}^{\wedge} 3$ \\
\hline 189 & Mass Flow of LN2 & $=B 186^{*} B 188^{*} 60$ & 4861 & $1 \mathrm{bm} / \mathrm{hr}$ \\
\hline
\end{tabular}

